\title{
Vectorial nonparaxial propagation equation in the presence of a tensorial refractive-index perturbation
}

\author{
Alessandro Ciattoni and Paolo Di Porto \\ Dipartimento di Fisica, Università dell'Aquila, 67010 L'Aquila, Italy, and Istituto Nazionale Fisica della Materia, \\ Unità di Roma I, Rome, Italy \\ Bruno Crosignani* and Amnon Yariv \\ Department of Applied Physics, California Institute of Technology, Pasadena, California 91125
}

Received August 5, 1999; revised manuscript received December 21, 1999

\begin{abstract}
The standard scalar paraxial parabolic (Fock-Leontovich) propagation equation is generalized to include allorder nonparaxial corrections in the significant case of a tensorial refractive-index perturbation on a homogeneous isotropic background. In the resultant equation, each higher-order nonparaxial term (associated with diffraction in homogeneous space and scaling as the ratio between beam waist and diffraction length) possesses a counterpart (associated with the refractive-index perturbation) that allows one to preserve the vectorial nature of the problem $(\nabla \nabla \cdot \mathbf{E} \neq 0)$. The tensorial character of the refractive-index variation is shown to play a particularly relevant role whenever the tensor elements $\delta n_{x z}$ and $\delta n_{y z}(z$ is the propagation direction) are not negligible. For this case, an application to elasto-optically induced optical activity and to nonlinear propagation in the presence of the optical Kerr effect is presented. (C) 2000 Optical Society of America [S0740-3224(00)00405-7]
\end{abstract}

OCIS codes: $260.0260,350.5500$.

\section{INTRODUCTION}

A difficulty in describing the nonparaxial propagation of an electromagnetic field $\mathbf{E}$ through an unbounded homogeneous or inhomogeneous medium by means of a parabolic wave equation arises whenever the beam waist $w_{0}$ and the diffraction length $d=k w_{0}^{2}$ (where $k$ is the radiation wave number) become comparable. In this situation, the reason for the failure of the parabolic equation description is twofold: First, the term that contains $\nabla_{\perp}{ }^{2} \mathbf{E}$ does not account completely for diffraction and, second, the assumption that $\nabla \nabla \cdot \mathbf{E}=0$ cannot be maintained any longer.

Starting from the pioneering research of Lax et al., ${ }^{1}$ and notwithstanding the conceptual and practical relevance of a problem that has been pointed out in many optical textbooks, ${ }^{2}$ few attempts have been made to generalize the parabolic wave equation in a rigorous way to include all-order corrections in the smallness parameter $\varepsilon=w_{0} / d=1 / k w_{0}$ while fully preserving the vectorial nature of the problem. To the best of our knowledge, the only simple approach to the problem has been provided, to the second order in $\varepsilon$ and for a scalar refractive-index distribution, by Savchencko and Zel'dovich, ${ }^{3}$ who adopted a straightforward iterative scheme. Actually, the necessity for being able to deal with a tensorial refractive-index distribution is not only conceptual but also practical. In fact, one of the relevant applications of a nonparaxial propagation equation concerns nonlinear optics, for which, typically, the nonlinear refractive index possesses a tensorial nature. In particular, the role of higher-order nonparaxial terms in the propagation equation that describes beam breakup owing to catastrophic collapse of self-focusing and, more generally, beam spatial evolution in the presence of the optical Kerr nonlinearity has been the object of a renewed interest in the past few years. ${ }^{4-9}$

In the present paper we are able to derive, under general assumptions, the propagation equation that describes the evolution of the electric field $\mathbf{E}$ in the presence of a refractive index consisting of a homogeneous part $n_{0}$ and a perturbative tensorial part $\delta \overleftrightarrow{\mathbf{n}}(\mathbf{r})$. This equation, which is first order in $\partial / \partial z$ (where $z$ is the propagation direction) accounts for all higher-order nonparaxial contributions through the presence of higher-order transverse derivatives of the field and is fully vectorial, in the sense that the condition $\nabla \nabla \cdot \mathbf{E}=0$ is never required. Remarkably, the lowest-order correction term to the standard parabolic equation turns out to be strictly associated with the tensorial nature of $\delta \overleftrightarrow{\mathbf{n}}(\mathbf{r})$ and is missing whenever $\delta \overleftrightarrow{\mathbf{n}}(\mathbf{r})$ is a scalar. In this respect, our equation provides the correct approach to bidimensional nonparaxial propagation in the presence of the nonlinear Kerr effect, because in this case $\delta \overleftrightarrow{\mathbf{n}}(\mathbf{r})$ is a tensor. ${ }^{10}$

\section{CONCEPT OF PARAXIALITY}

We recall briefly the derivation of the standard paraxial parabolic equation. The starting point is the vectorial Helmholtz equation obeyed by the monochromatic field, directly deduced from Maxwell's equations written for a scalar refractive-index distribution $n(\mathbf{r})$, which reads as 


$$
\begin{aligned}
\nabla^{2} \mathbf{E}-\nabla & \nabla \cdot \mathbf{E}+\frac{\omega^{2}}{c^{2}} n^{2}(\mathbf{r}) \mathbf{E} \\
& =\nabla^{2} \mathbf{E}+2 \nabla(\mathbf{E} \cdot \nabla \ln n)+\frac{\omega^{2}}{c^{2}} n^{2}(\mathbf{r}) \mathbf{E}=0 .
\end{aligned}
$$

We can then write $n^{2}(\mathbf{r})=\left[n_{0}+\delta n(\mathbf{r})\right]^{2} \cong n_{0}{ }^{2}$ $+2 n_{0} \delta n(\mathbf{r}), \quad k=\omega n_{0} / c, \quad$ and $\quad \mathbf{r}=\left(\mathbf{r}_{\perp}, \mathbf{z}\right), \quad \mathbf{E}(\mathbf{r}, t)$ $=\exp (i k z-i \omega t) \mathbf{A}\left(\mathbf{r}_{\perp}, z\right)$, separate the Laplacian into a transverse and a longitudinal part, $\nabla^{2}=\nabla_{\perp}^{2}+\partial^{2} / \partial z^{2}$, and take advantage of the slowly varying amplitude approximation (SVA) hypothesis according to which the longitudinal scale of variation of $\mathbf{A}\left(\mathbf{r}_{\perp}, z\right)$ is much larger than $1 / k$. The result, assuming that $\nabla \nabla \cdot \mathbf{E}=0$, is a parabolic equation in the standard form

$$
\left[i \partial / \partial z+(1 / 2 k) \nabla_{\perp}^{2}\right] \mathbf{A}\left(\mathbf{r}_{\perp}, z\right)=-\left(k / n_{0}\right) \delta n(\mathbf{r}) \mathbf{A}\left(\mathbf{r}_{\perp}, z\right) .
$$

Obviously, Eq. (2) is capable of describing only scalar propagation (different components of the field are uncoupled), and diffraction is accounted for through the presence of the term $(1 / 2 k) \nabla_{\perp}^{2} \mathbf{A}\left(\mathbf{r}_{\perp}, z\right)$ alone, this being the price that has to be paid for completely neglecting the term $\partial^{2} / \partial z^{2} \mathbf{A}\left(\mathbf{r}_{\perp}, z\right)$. As a consequence, Eq. (2) fails to describe propagation correctly as soon as the transverse beam waist and the diffraction length become comparable.

To formulate a nonparaxial propagation theory we have to give a precise meaning to the term paraxiality. To this end let us consider the standard angular spectrum solution of the scalar Helmholtz equation that describes monochromatic light propagation in a homogeneous medium:

$$
\mathbf{E}\left(\mathbf{r}_{\perp}, z\right)=\int \mathrm{d}^{2} \mathbf{k}_{\perp} \exp \left(i \mathbf{k}_{\perp} \cdot \mathbf{r}_{\perp}+i \sqrt{k^{2}-k_{\perp}{ }^{2}} z\right) \tilde{\mathbf{E}}\left(\mathbf{k}_{\perp}\right),
$$

which is the superposition of all the propagating and evanescent modes of unbounded space. In this simple case the beam described by Eq. (3) is termed paraxial if the field $\widetilde{\mathbf{E}}\left(\mathbf{k}_{\perp}\right)$ is practically nonvanishing only in a small portion of the $\left(k_{x}, k_{y}\right)$ plane whose diameter is much smaller than the wave number $k$, a situation in which the propagation directions of the plane waves in which the field is expanded are not too different from the $z$ axis. Accordingly, Eq. (3) can be approximated by

$$
\begin{aligned}
\mathbf{E}\left(\mathbf{r}_{\perp}, z\right)= & \exp (i k z) \int \mathrm{d}^{2} \mathbf{k}_{\perp} \\
& \times \exp \left[i \mathbf{k}_{\perp} \cdot \mathbf{r}_{\perp}-i(z / 2 k) k_{\perp}{ }^{2}\right] \widetilde{\mathbf{E}}\left(\mathbf{k}_{\perp}\right) \\
\equiv & \exp (i k z) \mathbf{A}\left(\mathbf{r}_{\perp}, z\right),
\end{aligned}
$$

where $\mathbf{A}\left(\mathbf{r}_{\perp}, z\right)$ satisfies the parabolic equation

$$
\left[i \partial / \partial z+(1 / 2 k) \nabla_{\perp}^{2}\right] \mathbf{A}\left(\mathbf{r}_{\perp}, z\right)=0,
$$

which shows the equivalence between paraxiality and the SVA.

In the general case of a light beam propagating in an inhomogeneous and anisotropic medium, simple expansion (3) does not apply, but the definition of paraxiality can be simply generalized from the previous definition.
In fact, the vanishing of the beam at infinity in the plane orthogonal to the propagation direction implies that each component of the electromagnetic field can be Fourier decomposed with respect to the transverse spatial coordinates. Thus, for any component $F\left(\mathbf{r}_{\perp}, z\right)$ of the field, it is possible to write

$$
F\left(\mathbf{r}_{\perp}, z\right)=\int \mathrm{d}^{2} \mathbf{k}_{\perp} \exp \left(i \mathbf{k}_{\perp} \cdot \mathbf{r}_{\perp}\right) \tilde{F}\left(\mathbf{k}_{\perp}, z\right) .
$$

We can now term a beam paraxial if the region $\Omega$ of the $\left(k_{x}, k_{y}\right)$ plane over which $\widetilde{F}\left(\mathbf{k}_{\perp}, z\right)$ is nonvanishing has a transverse extension much smaller than $k$, for all $z$. A possible smallness parameter $\varepsilon$ that characterizes paraxiality is then the ratio between the diameter of $\Omega$ and $k$, that is, $\varepsilon=\operatorname{diam}(\Omega) / k$. Using a well-known property of the Fourier analysis, we can relate $\operatorname{diam}(\Omega)$ to beam waist $w_{0}$ by $\operatorname{setting} \operatorname{diam}(\Omega) \cong 1 / w_{0}$, so $\varepsilon$ turns out to be the ratio between the beam waist and diffraction length $d$ $=k w_{0}^{2}$, that is, $\varepsilon=1 / k w_{0}$

\section{BEYOND THE PARAXIAL APPROXIMATION}

We wish to derive a theory of propagation that avoids the limitations contained in the standard paraxial theory. To this end we do not assume that $\nabla \nabla \cdot \mathbf{E}=0$ and we do not make use of the SVA. In fact, as we shall see, nonparaxiality couples propagation and polarization state, and this is incompatible with the condition $\nabla \nabla \cdot \mathbf{E}=0$; besides, the SVA gives only the lowest description of propagation and has to come from the theory in a natural way as the lowest order in $\varepsilon$. We start directly from firstorder Maxwell's equations for a monochromatic electromagnetic field, that is,

$$
\begin{aligned}
& \nabla \times \mathbf{E}=i \omega \mathbf{B}, \\
& \nabla \times \mathbf{B}=-i\left(\omega / c^{2}\right) \overleftrightarrow{\boldsymbol{\varepsilon}}: \mathbf{E},
\end{aligned}
$$

where $\overleftrightarrow{\boldsymbol{\varepsilon}}(\mathbf{r})$ represents the (relative) tensorial dielectric constant of the medium. From now on we define (with the standard sum convention over repeated indices) $(\overleftrightarrow{\mathbf{A}}: \overleftrightarrow{\mathbf{B}})_{i j}=A_{i k} B_{k j}, \quad(\overleftrightarrow{\mathbf{A}}: \mathbf{b})_{i}=A_{i j} b_{j}, \quad(\mathbf{a}: \mathbf{b})_{i j}=a_{i} b_{j}, \quad$ and $\mathbf{a} \cdot \mathbf{b}=a_{i} b_{i}$.

To begin with, we use a general property of the electromagnetic field that allows for the separability of transverse and longitudinal components of the electric and magnetic fields. More precisely, after writing $\nabla=\nabla_{\perp}$ $+\hat{\mathbf{z}} \partial / \partial z, \mathbf{E}=\mathbf{E}_{\perp}+\mathbf{E}_{z}$, and $\mathbf{B}=\mathbf{B}_{\perp}+\mathbf{B}_{z}$, we get (see Appendix A)

$$
\begin{aligned}
& \mathbf{B}_{z}=(1 / i \omega) \nabla_{\perp} \times \mathbf{E}_{\perp}, \\
& \mathbf{E}_{z}=\left(i c^{2} / \varepsilon_{z z} \omega\right) \nabla_{\perp} \times \mathbf{B}_{\perp}-\left(\mathbf{q} \cdot \mathbf{E}_{\perp} / \varepsilon_{z z}\right) \hat{\mathbf{z}},
\end{aligned}
$$

where the transverse vector $\mathbf{q}$ is defined as $\mathbf{q}=\left(\varepsilon_{z x} \hat{\mathbf{x}}\right.$ $\left.+\varepsilon_{z y} \hat{\mathbf{y}}\right)$. In turn, $\mathbf{E}_{\perp}$ and $\mathbf{B}_{\perp}$ obey the coupled system of equations (see Appendix A)

$$
\begin{aligned}
i \omega\left(\hat{\mathbf{z}} \times \partial \mathbf{B}_{\perp} / \partial z\right)= & \left(\omega^{2} / c^{2}\right) \overleftrightarrow{\boldsymbol{\varepsilon}}_{\perp}: \mathbf{E}_{\perp}-\nabla_{\perp} \times \nabla_{\perp} \times \mathbf{E}_{\perp} \\
& +\left(i \omega / \varepsilon_{z z}\right)\left(\hat{\mathbf{z}} \cdot \nabla_{\perp} \times \mathbf{B}_{\perp}\right) \mathbf{q} \\
& -\left(\omega^{2} / c^{2}\right)\left(\mathbf{q} \cdot \mathbf{E}_{\perp} / \varepsilon_{z z}\right) \mathbf{q}
\end{aligned}
$$




$$
\begin{aligned}
\left(\omega / i c^{2}\right)\left(\hat{\mathbf{z}} \times \partial \mathbf{E}_{\perp} / \partial z\right) & \\
= & \left(\omega^{2} / c^{2}\right) \mathbf{B}_{\perp}-\nabla_{\perp} \times\left[\left(\nabla_{\perp} \times \mathbf{B}_{\perp}\right) / \varepsilon_{z z}\right] \\
& +\left(\omega / i c^{2}\right) \nabla_{\perp} \times\left(\mathbf{q} \cdot \mathbf{E}_{\perp} / \varepsilon_{z z}\right) \hat{\mathbf{z}}, \quad
\end{aligned}
$$

where $\overleftrightarrow{\boldsymbol{\varepsilon}}_{\perp}$ is the transverse part of the dielectric tensor, that is, the $2 \times 2$ matrix obtained by $\overleftrightarrow{\boldsymbol{\varepsilon}}$ when the third row and the third column are dropped.

It is worth noting that Eqs. (9) and (10) contain only the transverse components of the electromagnetic field, and thus those equations suffice for determining that field. Once the transverse parts are known, Eqs. (8) provide the longitudinal components, so we can restrict our attention to Eqs. (9) and (10).

We now specialize our derivation to the case in which $\delta \overleftrightarrow{\mathbf{n}}(\mathbf{r})$ can be considered a small perturbation, which allows us to write approximately

$$
\begin{aligned}
\overleftrightarrow{\varepsilon} & =\left\lfloor n_{0}+\delta \overleftrightarrow{\mathbf{n}}(\mathbf{r})\right\rfloor^{2} \\
& \cong n_{0}{ }^{2}+2 n_{0} \delta \overleftrightarrow{\mathbf{n}}(\mathbf{r}), \\
\overleftrightarrow{\boldsymbol{\varepsilon}}_{\perp} & \cong n_{0}{ }^{2}+2 n_{0} \delta \overleftrightarrow{\mathbf{n}}_{\perp}(\mathbf{r}) ; \\
\mathbf{q} & \cong 2 n_{0} \delta \mathbf{n}^{\prime}=2 n_{0}\left(\delta n_{z x} \hat{\mathbf{x}}+\delta n_{z y} \hat{\mathbf{y}}\right) ; \\
\varepsilon_{z z} & \cong n_{0}{ }^{2}+2 n_{0} \delta n_{z z}, \\
1 / \varepsilon_{z z} & \cong 1 / n_{0}{ }^{2}-2 \delta n_{z z} / n_{0}{ }^{3} .
\end{aligned}
$$

After the above relations have been introduced into Eqs. (9) and (10), those equations can be rewritten as

$$
\begin{aligned}
\left(k^{2} / i \omega\right) & \left(\hat{\mathbf{z}} \times \partial \mathbf{E}_{\perp} / \partial z\right) \\
= & k^{2} \mathbf{B}_{\perp}-\nabla_{\perp} \times \nabla_{\perp} \times \mathbf{B}_{\perp}+\left(2 / n_{0}\right) \nabla_{\perp}\left(\delta n_{z z} \nabla_{\perp} \times \mathbf{B}_{\perp}\right) \\
& -\left(2 k^{2} / i \omega n_{0}\right) \hat{\mathbf{z}} \times \nabla_{\perp}\left(\delta \mathbf{n}^{\prime} \cdot \mathbf{E}_{\perp}\right),
\end{aligned}
$$

$i \omega\left(\hat{\mathbf{z}} \times \partial \mathbf{B}_{\perp} / \partial z\right)$

$$
\begin{aligned}
= & k^{2} \mathbf{E}_{\perp}-\nabla_{\perp} \times \nabla_{\perp} \times \mathbf{E}_{\perp}+2\left(k^{2} / n_{0}\right) \delta \overleftrightarrow{\mathbf{n}}_{\perp}: \mathbf{E}_{\perp} \\
& +\left(2 i \omega / n_{0}\right)\left(\hat{\mathbf{z}} \cdot \nabla_{\perp} \times \mathbf{B}_{\perp}\right) \delta \mathbf{n}^{\prime} .
\end{aligned}
$$

Inasmuch as the concept of paraxiality is simply stated in Fourier space, we Fourier transform both sides of Eqs. (15) and (16). After defining the Fourier transform $\widetilde{C}\left(\mathbf{k}_{\perp}, z\right)$ of a given function $C\left(\mathbf{r}_{\perp}, z\right)$ as

$$
\widetilde{C}\left(\mathbf{k}_{\perp}, z\right)=(1 / 2 \pi)^{2} \int \mathrm{d}^{2} \mathbf{r}_{\perp} \exp \left(-i \mathbf{k}_{\perp} \cdot \mathbf{r}_{\perp}\right) C\left(\mathbf{r}_{\perp}, z\right),
$$

we obtain (see Appendix B)

$$
\begin{aligned}
\frac{i k^{2}}{\omega} \frac{\partial \tilde{\mathbf{E}}_{\perp}}{\partial z}= & \overleftrightarrow{\boldsymbol{\sigma}}: \overleftrightarrow{\Lambda}: \tilde{\mathbf{B}}_{\perp} \\
& +\frac{2}{n_{0}} \int \mathrm{d}^{2} \mathbf{k}_{\perp}{ }^{\prime} \delta \tilde{n}_{z z}\left(\mathbf{k}_{\perp}-\mathbf{k}_{\perp}{ }^{\prime}, z\right)\left(\mathbf{k}_{\perp}: \mathbf{k}_{\perp}{ }^{\prime}\right) \\
& : \overleftrightarrow{\boldsymbol{\sigma}}: \tilde{\mathbf{B}}_{\perp}\left(\mathbf{k}_{\perp}, z\right)+\frac{2 k^{2}}{\omega n_{0}} \int \mathrm{d}^{2} \mathbf{k}_{\perp}{ }^{\prime} \\
& {\left[\mathbf{k}_{\perp}: \delta \tilde{\mathbf{n}}^{\prime}\left(\mathbf{k}_{\perp}-\mathbf{k}_{\perp}{ }^{\prime}, z\right)\right]: \tilde{\mathbf{E}}_{\perp}\left(\mathbf{k}_{\perp}{ }^{\prime}, z\right) }
\end{aligned}
$$

$$
\begin{aligned}
\frac{\omega}{i} \frac{\partial \widetilde{\mathbf{B}}_{\perp}}{\partial z}= & \overleftrightarrow{\boldsymbol{\sigma}}: \overleftrightarrow{\Lambda}: \widetilde{\mathbf{E}}_{\perp} \\
& +\frac{2 k^{2}}{n_{0}} \int \mathrm{d}^{2} \mathbf{k}_{\perp}{ }^{\prime} \overleftrightarrow{\boldsymbol{\sigma}}: \delta \widetilde{\mathbf{n}}_{\perp}\left(\mathbf{k}_{\perp}-\mathbf{k}_{\perp}{ }^{\prime}, z\right): \widetilde{\mathbf{E}}_{\perp}\left(\mathbf{k}_{\perp}{ }^{\prime}, z\right) \\
& -\frac{2 \omega}{n_{0}} \int \mathrm{d}^{2} \mathbf{k}_{\perp}{ }^{\prime} \overleftrightarrow{\boldsymbol{\sigma}}:\left[\delta \widetilde{\mathbf{n}}^{\prime}\left(\mathbf{k}_{\perp}-\mathbf{k}_{\perp}{ }^{\prime}, z\right): \mathbf{k}_{\perp}{ }^{\prime}\right] \\
& : \overleftrightarrow{\sigma}^{+}: \widetilde{\mathbf{B}}_{\perp}\left(\mathbf{k}_{\perp}{ }^{\prime}, z\right),
\end{aligned}
$$

where we have introduced the $2 \times 2$ matrices

$$
\begin{aligned}
& \overleftrightarrow{\boldsymbol{\sigma}}=\left[\begin{array}{cc}
0 & -1 \\
1 & 0
\end{array}\right], \quad \overleftrightarrow{\boldsymbol{\sigma}}^{+}=\left[\begin{array}{cc}
0 & 1 \\
-1 & 0
\end{array}\right], \\
& \overleftrightarrow{\boldsymbol{\Lambda}}=\left[\begin{array}{cc}
k^{2}-k_{y}{ }^{2} & k_{x} k_{y} \\
k_{x} k_{y} & k^{2}-k_{x}{ }^{2}
\end{array}\right] .
\end{aligned}
$$

We now look for solutions of Eqs. (18) and (19) of the form

$$
\begin{aligned}
\widetilde{\mathbf{E}}_{\perp}\left(\mathbf{k}_{\perp}, z\right)= & \widetilde{\mathbf{E}}_{\perp}{ }^{(+)}\left(\mathbf{k}_{\perp}, z\right) \exp (i h z) \\
& +\widetilde{\mathbf{E}}_{\perp}{ }^{(-)}\left(\mathbf{k}_{\perp}, z\right) \exp (-i h z), \\
\widetilde{\mathbf{B}}_{\perp}\left(\mathbf{k}_{\perp}, z\right)= & \left(\frac{k^{2} h}{\omega}\right): \overleftrightarrow{\Lambda}^{-1}: \overleftrightarrow{\boldsymbol{\sigma}}_{:}\left[\widetilde{\mathbf{E}}_{\perp}{ }^{(+)}\left(\mathbf{k}_{\perp}, z\right) \exp (i h z)\right. \\
& \left.-\widetilde{\mathbf{E}}_{\perp}{ }^{(-)}\left(\mathbf{k}_{\perp}, z\right) \exp (-i h z)\right],
\end{aligned}
$$

where $h=\sqrt{k^{2}-k_{\perp}^{2}}$, that describe the field as the superposition of two counterpropagating components, each undergoing diffraction. Inserting Eqs. (21) into Eqs. (18) and (19), we obtain (see Appendix C)

$$
\begin{aligned}
& \partial \widetilde{\mathbf{E}}_{\perp}{ }^{(+)} / \partial z=\left(i / n_{0}\right) \int \mathrm{d}^{2} \mathbf{k}_{\perp}{ }^{\prime} \stackrel{\boldsymbol{\Gamma}}{ }^{(+)}\left(\mathbf{k}_{\perp}, \mathbf{k}_{\perp}{ }^{\prime}, z\right) \\
& : \widetilde{\mathbf{E}}_{\perp}{ }^{(+)}\left(\mathbf{k}_{\perp}{ }^{\prime}, z\right) \exp \left[i\left(h^{\prime}-h\right) z\right]
\end{aligned}
$$

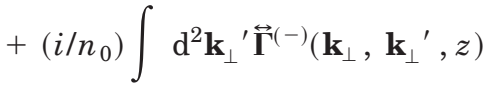

$$
\begin{aligned}
& : \tilde{\mathbf{E}}_{\perp}{ }^{(-)}\left(\mathbf{k}_{\perp}{ }^{\prime}, z\right) \exp \left[-i\left(h^{\prime}+h\right) z\right], \\
& \partial \widetilde{\mathbf{E}}_{\perp}{ }^{(-)} / \partial z=\left(i / n_{0}\right) \int \mathrm{d}^{2} \mathbf{k}_{\perp}{ }^{\prime} \overleftrightarrow{\Theta}^{(+)}\left(\mathbf{k}_{\perp}, \mathbf{k}_{\perp}{ }^{\prime}, z\right) \\
& : \tilde{\mathbf{E}}_{\perp}{ }^{(+)}\left(\mathbf{k}_{\perp}{ }^{\prime}, z\right) \exp \left[i\left(h^{\prime}+h\right) z\right] \\
& +\left(i / n_{0}\right) \int \mathrm{d}^{2} \mathbf{k}_{\perp}{ }^{\prime} \overleftrightarrow{\boldsymbol{\Theta}}^{(-)}\left(\mathbf{k}_{\perp}, \mathbf{k}_{\perp}{ }^{\prime}, z\right) \\
& : \widetilde{\mathbf{E}}_{\perp}{ }^{(-)}\left(\mathbf{k}_{\perp}{ }^{\prime}, z\right) \exp \left[i\left(h-h^{\prime}\right) z\right], \\
& \stackrel{\boldsymbol{\Gamma}}{( \pm)}^{( \pm}= \pm \delta \widetilde{n}_{z z}\left(\mathbf{k}_{\perp}-\mathbf{k}_{\perp}{ }^{\prime}, z\right)\left[\left(\mathbf{k}_{\perp}: \mathbf{k}_{\perp}{ }^{\prime}\right) / h^{\prime}\right] \\
& -\left[\mathbf{k}_{\perp}: \delta \widetilde{\mathbf{n}}^{\prime}\left(\mathbf{k}_{\perp}-\mathbf{k}_{\perp}{ }^{\prime}, z\right)\right] \\
& -\overleftrightarrow{\boldsymbol{\sigma}}: \overleftrightarrow{\Lambda}: \overleftrightarrow{\boldsymbol{\sigma}}:\left\{\delta \widetilde{\mathbf{n}}_{\perp}\left(\mathbf{k}_{\perp}-\mathbf{k}_{\perp}{ }^{\prime}, z\right) / h\right. \\
& \left.\mp\left[\delta \widetilde{\mathbf{n}}^{\prime}\left(\mathbf{k}_{\perp}-\mathbf{k}_{\perp}{ }^{\prime}, z\right): \mathbf{k}_{\perp}{ }^{\prime}\right] / h h^{\prime}\right\}
\end{aligned}
$$

and $\overleftrightarrow{\boldsymbol{\Theta}}^{( \pm)}$is obtained by $\overleftrightarrow{\boldsymbol{\Gamma}}^{( \pm)}$with the exchange $\overleftrightarrow{\Lambda}$ $\rightarrow-\overleftrightarrow{\Lambda}$. 
At this point, if we assume a small variation of the refractive index with respect to $z$ on a scale comparable with $1 / k$, it is possible to neglect coupling between forward and backward propagation fields (the possibility of accumulating effects of counterpropagating waves, including nonpropagating longitudinal ones, was studied in Refs. 11 and 12). Accordingly, Eqs. (22) and (23) decouple into two equations that describe $\widetilde{\mathbf{E}}_{\perp}{ }^{(+)}$and $\widetilde{\mathbf{E}}_{\perp}{ }^{(-)}$ separately. In particular, we can write

$$
\begin{aligned}
\partial \tilde{\mathbf{E}}_{\perp}{ }^{(+)} / \partial z= & \left(i / n_{0}\right) \int \mathrm{d}^{2} \mathbf{k}_{\perp}{ }^{\prime} \stackrel{\leftrightarrow}{\boldsymbol{\Gamma}}^{(+)}\left(\mathbf{k}_{\perp}, \mathbf{k}_{\perp}{ }^{\prime}, z\right) \\
& : \widetilde{\mathbf{E}}_{\perp}{ }^{(+)}\left(\mathbf{k}_{\perp}{ }^{\prime}, z\right) \exp \left[i\left(h^{\prime}-h\right) z\right],
\end{aligned}
$$

where, taking advantage of the relation $\overleftrightarrow{\boldsymbol{\sigma}}: \overleftrightarrow{\Lambda}: \overleftrightarrow{\boldsymbol{\sigma}}=-k^{2}$ $+\mathbf{k}_{\perp}: \mathbf{k}_{\perp}$,

$$
\begin{aligned}
\overleftrightarrow{\boldsymbol{\Gamma}}^{(+)}= & \delta \widetilde{n}_{z z}\left(\mathbf{k}_{\perp}-\mathbf{k}_{\perp}{ }^{\prime}, z\right)\left[\left(\mathbf{k}_{\perp}: \mathbf{k}_{\perp}{ }^{\prime}\right) / h^{\prime}\right] \\
& -\left[\mathbf{k}_{\perp}: \delta \widetilde{\mathbf{n}}^{\prime}\left(\mathbf{k}_{\perp}-\mathbf{k}_{\perp}{ }^{\prime}, z\right)\right]+\left[k^{2}-\left(\mathbf{k}_{\perp}: \mathbf{k}_{\perp}\right)\right] \\
& :\left\{\delta \widetilde{\mathbf{n}}_{\perp}\left(\mathbf{k}_{\perp}-\mathbf{k}_{\perp}{ }^{\prime}, z\right) / h\right. \\
& \left.-\left[\delta \widetilde{\mathbf{n}}^{\prime}\left(\mathbf{k}_{\perp}-\mathbf{k}_{\perp}{ }^{\prime}, z\right): \mathbf{k}_{\perp}{ }^{\prime}\right] / h h^{\prime}\right\} .
\end{aligned}
$$

Equation (25) completely describes the evolution of the forward propagating beam in Fourier space. It is not difficult to derive, starting from Eq. (25), the equation of evolution in ordinary space (see Appendix D). It reads as

$$
\begin{aligned}
& \left(i \frac{\partial}{\partial z}+\sqrt{k^{2}+\nabla_{\perp}^{2}}\right) \mathbf{E}_{\perp}{ }^{(+)} \\
& =-\frac{k^{2}}{n_{0}} \frac{1}{\sqrt{k^{2}+\nabla_{\perp}^{2}}}\left(\delta \overleftrightarrow{\mathbf{n}}_{\perp}: \mathbf{E}_{\perp}{ }^{(+)}\right) \\
& +\frac{1}{n_{0}} \nabla_{\perp}\left[\delta n_{z z} \frac{1}{\sqrt{k^{2}+\nabla_{\perp}^{2}}}\left(\nabla_{\perp} \cdot \mathbf{E}_{\perp}{ }^{(+)}\right)\right] \\
& -\frac{1}{n_{0}} \frac{1}{\sqrt{k^{2}+\nabla_{\perp}^{2}}} \nabla_{\perp}\left[\nabla_{\perp} \cdot\left(\delta \overleftrightarrow{\mathbf{n}}_{\perp}: \mathbf{E}_{\perp}^{(+)}\right)\right] \\
& -\frac{i}{n_{0}} \nabla_{\perp}\left[\delta \mathbf{n}^{\prime} \cdot \mathbf{E}_{\perp}{ }^{++)}\right]-\frac{i k^{2}}{n_{0}} \frac{1}{\sqrt{k^{2}+\nabla_{\perp}^{2}}} \\
& {\left[\delta \mathbf{n}^{\prime} \frac{1}{\sqrt{k^{2}+\nabla_{\perp}^{2}}}\left(\nabla_{\perp} \cdot \mathbf{E}_{\perp}{ }^{(+)}\right)\right]-\frac{i}{n_{0}} \frac{1}{\sqrt{k^{2}+\nabla_{\perp}^{2}}} \nabla_{\perp}} \\
& \left\{\nabla_{\perp} \cdot\left[\delta \mathbf{n}^{\prime} \frac{1}{\sqrt{k^{2}+\nabla_{\perp}^{2}}}\left(\nabla_{\perp} \cdot \mathbf{E}_{\perp}^{(+)}\right)\right]\right\},
\end{aligned}
$$

which is the fundamental result of the present paper.

Despite its evident analytical complexity, Eq. (27) possesses some interesting features that are worth analyz- ing. First, it is completely equivalent to Maxwell equations and thus is capable of describing nonparaxial propagation to any order in $\varepsilon$ : In fact, the only approximation that we used to derive it was to neglect coupling between forward and backward propagating fields and to exploit the smallness of the refractive-index perturbation to neglect its square value (this last assumption is not really necessary and can easily be removed). Besides, it is first order in $\partial / \partial z$, whereas all the other differential operators involve transverse coordinates (a mathematical structure that is particularly suitable for numerical analysis); this circumstance confirms the redundancy of the SVA hypothesis that is usually invoked to reduce the second-order (in $\partial / \partial z$ ) Helmholtz equation to a first-order equation. $^{13}$

The above procedure can be generalized to include coupling with counterpropagating field $\mathbf{E}_{\perp}{ }^{(-)}$. The resultant coupled system of equations that describe the evolution of both $\mathbf{E}_{\perp}^{(+)}$and $\mathbf{E}_{\perp}^{(-)}$is given in Appendix E.

We note that, although Eq. (27) is formally equivalent to Maxwell's equations, the operators $\sqrt{k^{2}+\nabla_{\perp}^{2}}$ and $1 / \sqrt{k^{2}+\nabla_{\perp}^{2}}$ can be given a precise meaning through the relations

$$
\begin{aligned}
& \sqrt{k^{2}+\nabla_{\perp}^{2}} f\left(\mathbf{r}_{\perp}, z\right) \\
& \quad=\int \mathrm{d}^{2} \mathbf{k}_{\perp} \exp \left(i \mathbf{k}_{\perp} \cdot \mathbf{r}_{\perp}\right) \sqrt{k^{2}-k_{\perp}{ }^{2}} \tilde{f}\left(\mathbf{k}_{\perp}, z\right),
\end{aligned}
$$

$$
\begin{aligned}
& \frac{1}{\sqrt{\mathbf{k}^{2}+\nabla_{\perp}^{2}}} f\left(\mathbf{r}_{\perp}, z\right) \\
& \quad=\int \mathrm{d}^{2} \mathbf{k}_{\perp} \exp \left(i \mathbf{k}_{\perp} \cdot \mathbf{r}_{\perp}\right) \frac{1}{\sqrt{k^{2}-k_{\perp}^{2}}} \tilde{f}\left(\mathbf{k}_{\perp}, z\right),
\end{aligned}
$$

where $\tilde{f}\left(\mathbf{k}_{\perp}, z\right)$ is a well-behaved function of $\mathbf{k}_{\perp}$

\section{DERIVATION OF APPROXIMATE NONPARAXIAL EQUATIONS}

Let us first consider the linear case for which $\delta \overleftrightarrow{\mathbf{n}}$ is independent of field $\mathbf{E}$, and let us assume that $\delta \overleftrightarrow{\mathbf{n}}$ possesses a typical transverse scale of variation $l$ such that $l>w_{0}$. In this case, Eq. (27) contains two independent smallness parameters, i.e., $\varepsilon=1 / k w_{0}$ and $\delta$; the latter corresponds to the amplitude of the refractive-index variation that is relevant to the situation under consideration. Accordingly, the standard parabolic equation [see Eq. (2)] corresponds to keeping in Eq. (27) only the terms up to $\varepsilon^{2}$ on its left-hand side and to $\delta$ on its right-hand side. If the refractive-index variation tensor $\delta \overleftrightarrow{\mathbf{n}}$ is such that $\delta \mathbf{n}^{\prime}$ $=0$, then the first significant correction to the parabolic equation is of the order $\left|\delta n_{\perp}\right| \varepsilon^{2}$, whereas if $\delta \mathbf{n}^{\prime}$ is not vanishing, the correction is of the order $\left|\delta \mathbf{n}^{\prime}\right| \varepsilon$. In this approximation, after $\mathbf{A}_{\perp}=\mathbf{E}_{\perp} \exp (i k z)$ is introduced in the usual way and the relevant transverse operators are expanded as 


$$
\begin{aligned}
& \sqrt{k^{2}+\nabla_{\perp}^{2}}=k+\frac{1}{2 k} \nabla_{\perp}^{2}-\frac{1}{8 k^{3}} \nabla_{\perp}^{2} \nabla_{\perp}^{2}+\ldots \leftrightarrow \sqrt{k^{2}-k_{\perp}{ }^{2}}=k-\frac{1}{2 k} k_{\perp}{ }^{2}-\frac{1}{8 k^{3}} k_{\perp}{ }^{4}+\ldots \\
& \frac{1}{\sqrt{k^{2}+\nabla_{\perp}^{2}}}=\frac{1}{k}-\frac{1}{2 k^{3}} \nabla_{\perp}^{2}+\frac{3}{8 k^{3}} \nabla_{\perp}{ }^{2} \nabla_{\perp}{ }^{2}+\ldots \leftrightarrow \frac{1}{\sqrt{k^{2}-k_{\perp}^{2}}}=\frac{1}{k}+\frac{1}{2 k^{3}} k_{\perp}{ }^{2}+\frac{3}{8 k^{3}} k_{\perp}{ }^{4}+\ldots
\end{aligned}
$$

[for the meaning of operator expansion and truncation to a given order, see Eqs. (28) and (29)], Eq. (27) reduces to

$$
\begin{aligned}
& \left(i \frac{\partial}{\partial z}+\frac{1}{2 k} \nabla_{\perp}^{2}\right) \mathbf{A}_{\perp} \\
& =-\frac{k}{n_{0}} \delta \overleftrightarrow{\mathbf{n}}_{\perp}: \mathbf{A}_{\perp}-\frac{i}{n_{0}} \nabla_{\perp}\left(\delta \mathbf{n}^{\prime} \cdot \mathbf{A}_{\perp}\right) \\
& -\frac{i}{n_{0}}\left[\delta \mathbf{n}^{\prime}\left(\nabla_{\perp} \cdot \mathbf{A}_{\perp}\right)\right]+\frac{1}{2 n_{0} k} \nabla_{\perp}^{2}\left(\delta \overleftrightarrow{\mathbf{n}}_{\perp}: \mathbf{A}_{\perp}\right) \\
& +\frac{1}{n_{0} k} \nabla_{\perp}\left[\delta n_{z z}\left(\nabla_{\perp} \cdot \mathbf{A}_{\perp}\right)\right] \\
& -\frac{1}{n_{0} k} \nabla_{\perp}\left[\nabla_{\perp} \cdot\left(\delta \overleftrightarrow{\mathbf{n}}_{\perp}: \mathbf{A}_{\perp}\right)\right] .
\end{aligned}
$$

Note that, in the scalar case, Eq. (31) takes the simple form

$$
\begin{aligned}
\left(i \frac{\partial}{\partial z}+\frac{1}{2 k} \nabla_{\perp}^{2}\right) \mathbf{A}_{\perp}= & -\frac{k}{n_{0}} \delta n \mathbf{A}_{\perp}+\frac{1}{2 n_{0} k} \nabla_{\perp}^{2}\left(\delta n \mathbf{A}_{\perp}\right) \\
& -\frac{1}{n_{0} k} \nabla_{\perp}\left(\mathbf{A}_{\perp} \cdot \nabla_{\perp} \delta n\right),
\end{aligned}
$$

which coincides with the one worked out in Ref. 3 [see Eq. degenerate guided modes of orthogonal polarization. In this case, the dominant interaction terms are those that contain $\delta \mathbf{n}^{\prime}$; besides, the (diffraction) terms that contain the transverse derivatives of $\mathbf{A}_{\perp}$ can, because of the very nature of the guided modes, be put equal to zero. Proceeding in this way, and indicating with $a_{x}(z)$ and $a_{y}(z)$ the amplitudes of the two orthogonal linearly polarized modes, we get

$$
\begin{aligned}
& i \frac{\mathrm{d}}{\mathrm{d} z} a_{x}=-k \frac{\delta n}{n_{0}} a_{x}+i \eta a_{y}, \\
& i \frac{\mathrm{d}}{\mathrm{d} z} a_{y}=-k \frac{\delta n}{n_{0}} a_{y}-i \eta a_{x},
\end{aligned}
$$

where $\delta n=\delta n_{x x}=\delta n_{y y}$ and $\eta=p n_{0}{ }^{2} \tau / 2$. We immediately check, after introducing the amplitudes $R=\left(a_{x}\right.$ $\left.-i a_{y}\right) / \sqrt{2}$ and $L=\left(a_{x}+i a_{y}\right) / \sqrt{2}$ of the right and left circularly polarized states, respectively, that the set of Eqs. (33) describes circular birefringes (optical activity).

In the case of nonlinear propagation, the two smallness parameters $\varepsilon$ and $\delta$ are no longer independent, and one has to consider each situation separately. As a relevant example, we describe below propagation in a homogeneous medium in the presence of the optical Kerr effect. In this case, the nonlinear refractive-index tensor has the form ${ }^{10}$

$$
\delta \overleftrightarrow{\mathbf{n}}=\frac{2}{3} n_{2}\left[\begin{array}{ccc}
|\mathbf{E}|^{2}+1 / 2\left|E_{x}\right|^{2} & 1 / 2 E_{y} E_{x}{ }^{*} & 1 / 2 E_{z} E_{x}{ }^{*} \\
1 / 2 E_{x} E_{y}{ }^{*} & |\mathbf{E}|^{2}+1 / 2\left|E_{y}\right|^{2} & 1 / 2 E_{z} E_{y}{ }^{*} \\
1 / 2 E_{x} E_{z}{ }^{*} & 1 / 2 E_{y} E_{z}{ }^{*} & |\mathbf{E}|^{2}+1 / 2\left|E_{z}\right|^{2}
\end{array}\right],
$$

(12) of Ref. 3].

In inspecting Eq. (31) it is worthwhile to remark on the appearance of the two terms that contain $\delta \mathbf{n}^{\prime}$, which would be completely missing for a diagonal (in particular, a scalar) refractive-index variation $\delta \overleftrightarrow{\mathbf{n}}$. In fact, if the nondiagonal elements $\delta n_{x z}$ and $\delta n_{y z}$ are comparable with $\delta n_{x x}, \delta n_{y y}$, and $\delta n_{z z}$, these terms turn out to be dominant with respect to the succesive terms, a circumstance that, to the best of our knowledge, was completely unnoticed until now.

As an example, let us consider elasto-optically induced optical activity in a twisted (about the axial $z$ direction) single-mode optical fiber. In this case, $\delta n_{x z}=p n_{0}{ }^{3} \tau y / 2$ and $\delta n_{y z}=p n_{0}{ }^{3} \tau x / 2$, where $p$ is the appropriate component of the elasto-optic tensor and $\tau$ is the twist rate. ${ }^{14}$ Let us specialize Eq. (31), which can be used also for guided propagation, to a single-mode fiber supporting two so

$$
\begin{aligned}
\delta \overleftrightarrow{\mathbf{n}}_{\perp} & =2 / 3 n_{2}\left|\mathbf{E}_{\perp}\right|^{2}+2 / 3 n_{2}\left|E_{z}\right|^{2}+1 / 3 n_{2}\left(\mathbf{E}_{\perp} * \mathbf{E}_{\perp}\right), \\
\delta \mathbf{n}^{\prime} & =1 / 3 n_{2} E_{z} \mathbf{E}_{\perp}{ }^{*} \\
\delta n_{z z} & =2 / 3 n_{2}\left|\mathbf{E}_{\perp}\right|^{2}+n_{2}\left|E_{z}\right|^{2},
\end{aligned}
$$

where $n_{2}$ is the so-called nonlinear refractive-index coefficient.

Because of the appearance of $E_{z}$ in the expression of $\delta \overleftrightarrow{\mathbf{n}}$, we need to express the longitudinal part of the field in terms of the transverse part. We can do this by taking advantage of the smallness of the nonlinear contribution $\left(n_{2}|\mathbf{E}|^{2} \ll 1\right)$, thus getting (see Appendix E) $E_{z}$ $\cong(i / k) \nabla_{\perp} \cdot \mathbf{E}_{\perp}$. Inserting this relation and Eq. (34) into Eq. (31) and neglecting terms with more than two 
$\nabla_{\perp}$, we obtain, after taking advantage of the identity $\left(\mathbf{A}_{\perp}^{*}: \mathbf{A}_{\perp}\right): \mathbf{A}_{\perp}=\left(\mathbf{A}_{\perp} \cdot \mathbf{A}_{\perp}\right) \mathbf{A}_{\perp} *$, the following equation:

$$
\begin{aligned}
\left(i \frac{\partial}{\partial z}+\right. & \left.\frac{1}{2 k} \nabla_{\perp}^{2}\right) \mathbf{A}_{\perp} \\
= & -\frac{2 k}{3} \frac{n_{2}}{n_{0}}\left|\mathbf{A}_{\perp}\right|^{2} \mathbf{A}_{\perp}-\frac{2}{3 k} \frac{n_{2}}{n_{0}}\left|\nabla_{\perp} \cdot \mathbf{A}_{\perp}\right|^{2} \mathbf{A}_{\perp} \\
& -\frac{k}{3} \frac{n_{2}}{n_{0}}\left(\mathbf{A}_{\perp} \cdot \mathbf{A}_{\perp}\right) \mathbf{A}_{\perp} *+\frac{1}{k} \frac{n_{2}}{n_{0}} \nabla_{\perp}\left[\left(\nabla_{\perp} \cdot \mathbf{A}_{\perp}\right)\left|\mathbf{A}_{\perp}\right|^{2}\right] \\
& +\frac{1}{3 k} \frac{n_{2}}{n_{0}}\left(\nabla_{\perp} \cdot \mathbf{A}_{\perp}\right)^{2} \mathbf{A}_{\perp} *+\frac{n_{2}}{3 n_{0} k} \nabla_{\perp}{ }^{2}\left(\left.\mathbf{A}_{\perp}\right|^{2} \mathbf{A}_{\perp}\right) \\
& +\frac{n_{2}}{6 n_{0} k} \nabla_{\perp}^{2}\left[\left(\mathbf{A}_{\perp} \cdot \mathbf{A}_{\perp}\right) \mathbf{A}_{\perp} *\right] \\
& -\frac{2}{3 k} \frac{n_{2}}{n_{0}} \nabla_{\perp}\left[\nabla_{\perp} \cdot\left(\left|\mathbf{A}_{\perp}\right|^{2} \mathbf{A}_{\perp}\right)\right] \\
& -\frac{1}{3 k} \frac{n_{2}}{n_{0}} \nabla_{\perp}\left\{\nabla_{\perp} \cdot\left[\left(\mathbf{A}_{\perp} \cdot \mathbf{A}_{\perp}\right) \mathbf{A}_{\perp} *\right]\right\}
\end{aligned}
$$

which describes the nonlinear vectorial nonparaxial evolution of the field in the presence of the optical Kerr effect.

\section{CONCLUSIONS}

Starting directly from Maxwell's equations, we have been able to generalize the standard paraxial approach to optical propagation in a medium described by a tensorial refractive-index variation $\delta \mathbf{n}$ over a scalar isotropic homogeneous background. More precisely, we have derived a partial differential equation, to first order in $\partial / \partial z$, describing forward propagation to every order in the ratio $\varepsilon=1 / k w_{0}$ between the beam wavelength and its waist, without introducing the transversality condition $\nabla \cdot \mathbf{E}$ $=0$ and thus preserving the full vectorial nature of the problem. In particular, the tensorial character of the refractive-index perturbation is shown to play a relevant role in that, whenever the elements $\delta n_{x z}$ and $\delta n_{y z}(z$ is the main propagation direction) become comparable to the transverse elements, the first correction to the parabolic paraxial equation is of the order of $\varepsilon$ and not of $\varepsilon^{2}$ as in the scalar case. As a simple application in the framework of linear optics, we have derived the equation that describes elasto-optically induced optical activity in a twisted single-mode optical fiber. In the frame-work of nonlinear optics, we have specialized our equation to the case of an intensity-dependent refractive index (optical Kerr effect) and obtained a fully vectorial propagation equation that contains nonparaxial contributions up to second order.

\section{APPENDIX A: SEPARATION OF LONGITUDINAL AND TRANSVERSE PARTS OF THE FIELD}

By use of the vector identities that are valid for any vector field $\mathbf{F}=\mathbf{F}_{\perp}+\mathbf{F}_{z}$ (with $\mathbf{F}_{\perp}=\mathbf{F}_{x} \hat{\mathbf{x}}+F_{y} \hat{\mathbf{y}}$ and $\mathbf{F}_{z}=F_{z} \hat{\mathbf{z}}$ ),

$$
(\nabla \times \mathbf{F})_{\perp}=\nabla_{\perp} \times \mathbf{F}_{z}+\hat{\mathbf{z}} \times \frac{\partial \mathbf{F}_{\perp}}{\partial z}(\nabla \times \mathbf{F})_{z}=\nabla_{\perp} \times \mathbf{F}_{\perp}
$$

it is straightforward to obtain from Maxwell's equations [Eqs. (9) and (10)]

$$
\begin{aligned}
& \nabla_{\perp} \times \mathbf{E}_{z}+\hat{\mathbf{z}} \times \frac{\partial \mathbf{E}_{\perp}}{\partial z}=i \omega \mathbf{B}_{\perp}, \\
& \nabla_{\perp} \times \mathbf{E}_{\perp}=i \omega \mathbf{B}_{z} ; \\
& \nabla_{\perp} \times \mathbf{B}_{z}+\hat{\mathbf{z}} \times \frac{\partial \mathbf{B}_{\perp}}{\partial z}=-\frac{i \omega}{c^{2}} \overleftrightarrow{\boldsymbol{\varepsilon}}_{\perp}: \mathbf{E}_{\perp}-\frac{i \omega}{c^{2}}\left(\hat{\mathbf{z}} \cdot \mathbf{E}_{z}\right) \mathbf{q} \\
& \nabla_{\perp} \times \mathbf{B}_{\perp}=-\frac{i \omega}{c^{2}}\left(\mathbf{q} \cdot \mathbf{E}_{\perp}\right) \hat{\mathbf{z}}-\frac{i \omega}{c^{2}} \varepsilon_{z z} \mathbf{E}_{z}
\end{aligned}
$$

where $\mathbf{q}=\left(\varepsilon_{z x} \hat{\mathbf{x}}+\varepsilon_{z y} \hat{\mathbf{y}}\right)$ and

$$
\overleftrightarrow{\boldsymbol{\varepsilon}}_{\perp}=\left[\begin{array}{ll}
\varepsilon_{x x} & \varepsilon_{x y} \\
\varepsilon_{y x} & \varepsilon_{y y}
\end{array}\right] .
$$

The longitudinal components of the fields can now be simply related to the transverse components. In fact, from Eqs. (A2.2) and (A3.2) we obtain

$$
\begin{aligned}
& \mathbf{B}_{z}=\frac{1}{i \omega} \nabla_{\perp} \times \mathbf{E}_{\perp}, \\
& \mathbf{E}_{z}=\frac{i c^{2}}{\varepsilon_{z z} \omega} \nabla_{\perp} \times \mathbf{B}_{\perp}-\frac{\mathbf{q} \cdot \mathbf{E}_{\perp}}{\varepsilon_{z z}} \hat{\mathbf{z}},
\end{aligned}
$$

which, inserted in Eqs. (A2.1) and (A3.1), respectively, yield

$$
\begin{aligned}
i \omega\left(\hat{\mathbf{z}} \times \partial \mathbf{B}_{\perp} / \partial z\right)= & \left(\omega^{2} / c^{2}\right) \overleftrightarrow{\boldsymbol{\varepsilon}}_{\perp}: \mathbf{E}_{\perp}-\nabla_{\perp} \times \nabla_{\perp} \times \mathbf{E}_{\perp} \\
& +\left(i \omega / \varepsilon_{z z}\right)\left(\hat{\mathbf{z}} \cdot \nabla_{\perp} \times \mathbf{B}_{\perp}\right) \mathbf{q} \\
& -\left(\omega^{2} / c^{2}\right)\left(\mathbf{q} \cdot \mathbf{E}_{\perp} / \varepsilon_{z z}\right) \mathbf{q}, \quad(\mathrm{A} 6) \\
\left(\omega / i c^{2}\right)\left(\hat{\mathbf{z}} \times \partial \mathbf{E}_{\perp} / \partial z\right)= & \left(\omega^{2} / c^{2}\right) \mathbf{B}_{\perp}-\nabla_{\perp}\left[\left(\nabla_{\perp} \times \mathbf{B}_{\perp}\right) / \varepsilon_{z z}\right] \\
& +\left(\omega / i c^{2}\right) \nabla_{\perp} \times\left(\mathbf{q} \cdot \mathbf{E}_{\perp} / \varepsilon_{z z}\right) \hat{\mathbf{z}} .
\end{aligned}
$$

\section{APPENDIX B: FOURIER TRANSFORM OF MAXWELL'S EQUATIONS FOR THE TRANSVERSE FIELD COMPONENT}

Fourier transforming Eq. (15) yields

$$
\begin{aligned}
\frac{k^{2}}{i \omega} \stackrel{\leftrightarrow}{\boldsymbol{\sigma}}: \frac{\partial \tilde{\mathbf{E}}_{\perp}}{\partial z}= & \overleftrightarrow{\Lambda}: \widetilde{\mathbf{B}}_{\perp}+\frac{2}{n_{0}} \frac{1}{(2 \pi)^{2}} \int \mathrm{d}^{2} \mathbf{r}_{\perp} \\
& \exp \left(-i \mathbf{k}_{\perp} \cdot \mathbf{r}_{\perp}\right) \nabla_{\perp} \times\left(\delta n_{z z} \nabla_{\perp} \times \mathbf{B}_{\perp}\right) \\
& -\frac{2 k^{2}}{i \omega n_{0}} \frac{1}{(2 \pi)^{2}} \int \mathrm{d}^{2} \mathbf{r}_{\perp} \exp \left(-i \mathbf{k}_{\perp} \cdot \mathbf{r}_{\perp}\right) \hat{\mathbf{z}} \\
& \times \nabla_{\perp}\left(\delta \mathbf{n}^{\prime} \cdot \mathbf{E}_{\perp}\right),
\end{aligned}
$$


where the $2 \times 2$ matrices $\overleftrightarrow{\sigma}$ and $\overleftrightarrow{\Lambda}$ are defined in Eq. (19). The two integrals on the right-hand side of Eq. (B1) can be rewritten by use of the well-known properties of the Fourier transform

$$
\begin{aligned}
& \int \frac{\mathrm{d}^{2} \mathbf{r}_{\perp}}{(2 \pi)^{2}} \exp \left(-i \mathbf{k}_{\perp} \cdot \mathbf{r}_{\perp}\right) \nabla_{\perp} \times\left(\delta n_{z z} \nabla_{\perp} \times \mathbf{B}_{\perp}\right) \\
& =i \mathbf{k}_{\perp} \times \int \frac{\mathrm{d}^{2} \mathbf{r}_{\perp}}{(2 \pi)^{2}} \exp \left(-i \mathbf{k}_{\perp} \cdot \mathbf{r}_{\perp}\right)\left(\delta n_{z z} \nabla_{\perp} \times \mathbf{B}_{\perp}\right) \\
& =i \mathbf{k}_{\perp} \times \int \mathrm{d}^{2} \mathbf{k}_{\perp}{ }^{\prime} \delta \widetilde{n}_{z z}\left(\mathbf{k}_{\perp}-\mathbf{k}_{\perp}{ }^{\prime}, z\right) \\
& \frac{1}{(2 \pi)^{2}} \int \mathrm{d}^{2} \mathbf{r}_{\perp} \exp \left(i \mathbf{k}_{\perp}{ }^{\prime} \cdot \mathbf{r}_{\perp}\right) \nabla_{\perp} \times \mathbf{B}_{\perp} \\
& =-\int \mathrm{d}^{2} \mathbf{k}_{\perp}{ }^{\prime} \delta \widetilde{n}_{z z}\left(\mathbf{k}_{\perp}-\mathbf{k}_{\perp}{ }^{\prime}, z\right) \mathbf{k}_{\perp} \times \mathbf{k}_{\perp}{ }^{\prime} \\
& \times \widetilde{\mathbf{B}}_{\perp}\left(\mathbf{k}_{\perp}{ }^{\prime}, z\right)=-\int \mathrm{d}^{2} \mathbf{k}_{\perp}{ }^{\prime} \delta \widetilde{n}_{z z}\left(\mathbf{k}_{\perp}-\mathbf{k}_{\perp}{ }^{\prime}, z\right) \\
& {\left[\overleftrightarrow{\boldsymbol{\sigma}}:\left(\mathbf{k}_{\perp}: \mathbf{k}_{\perp}{ }^{\prime}\right): \overleftrightarrow{\boldsymbol{\sigma}}\right]: \widetilde{\mathbf{B}}_{\perp}\left(\mathbf{k}_{\perp}{ }^{\prime}, z\right)} \\
& \int \frac{\mathrm{d}^{2} \mathbf{r}_{\perp}}{(2 \pi)^{2}} \exp \left(-i \mathbf{k}_{\perp} \cdot \mathbf{r}_{\perp}\right) \hat{\mathbf{z}} \times \nabla_{\perp}\left(\delta \mathbf{n}^{\prime} \cdot \mathbf{E}_{\perp}\right) \\
& =i \hat{\mathbf{z}} \times \mathbf{k}_{\perp} \frac{1}{(2 \pi)^{2}} \int \mathrm{d}^{2} \mathbf{r}_{\perp} \exp \left(-i \mathbf{k}_{\perp} \cdot \mathbf{r}_{\perp}\right) \delta \mathbf{n}^{\prime} \cdot \mathbf{E}_{\perp} \\
& =i \hat{\mathbf{z}} \times \mathbf{k}_{\perp} \int \mathrm{d}^{2} \mathbf{k}_{\perp}{ }^{\prime} \delta \widetilde{\mathbf{n}}^{\prime}\left(\mathbf{k}_{\perp}-\mathbf{k}_{\perp}{ }^{\prime}, z\right) \cdot \widetilde{\mathbf{E}}_{\perp}\left(\mathbf{k}_{\perp}{ }^{\prime}, z\right) \\
& =i \int \mathrm{d}^{2} \mathbf{k}_{\perp}{ }^{\prime} \stackrel{\leftrightarrow}{\sigma}: \mathbf{k}_{\perp}\left[\delta \widetilde{\mathbf{n}}^{\prime}\left(\mathbf{k}_{\perp}-\mathbf{k}_{\perp}{ }^{\prime}, z\right) \cdot \widetilde{\mathbf{E}}_{\perp}\left(\mathbf{k}_{\perp}{ }^{\prime}, z\right)\right] \\
& =i \int \mathrm{d}^{2} \mathbf{k}_{\perp}{ }^{\prime} \stackrel{\leftrightarrow}{\sigma}:\left[\mathbf{k}_{\perp}: \delta \widetilde{\mathbf{n}}^{\prime}\left(\mathbf{k}_{\perp}-\mathbf{k}_{\perp}{ }^{\prime}, z\right)\right]: \tilde{\mathbf{E}}_{\perp}\left(\mathbf{k}_{\perp}{ }^{\prime}, z\right) .
\end{aligned}
$$

Inserting Eqs. (B2) and (B3) into Eq. (B1) and multiplying the resultant equation by matrix $\overleftrightarrow{\boldsymbol{\sigma}}$, we obtain Eq. (17).

Proceeding in the same way for Eq. (15), we get

$$
\begin{aligned}
i \omega \overleftrightarrow{\boldsymbol{\sigma}}: \frac{\partial \widetilde{\mathbf{B}}}{\partial z}= & \overleftrightarrow{\Lambda}: \widetilde{\mathbf{E}}_{\perp}+2 \frac{k^{2}}{n_{0}} \\
& \int \mathrm{d}^{2} \mathbf{k}_{\perp}^{\prime} \delta \widetilde{\widetilde{\mathbf{n}}}_{\perp}\left(\mathbf{k}_{\perp}-\mathbf{k}_{\perp}{ }^{\prime}, z\right): \widetilde{\mathbf{E}}_{\perp}\left(\mathbf{k}_{\perp}^{\prime}, z\right) \\
& +2 \frac{i \omega}{n_{0}} \int \frac{\mathrm{d}^{2} \mathbf{r}_{\perp}}{(2 \pi)^{2}} \exp \left(-i \mathbf{k}_{\perp} \cdot \mathbf{r}_{\perp}\right) \\
& \left(\hat{\mathbf{z}} \cdot \nabla_{\perp} \times \mathbf{B}_{\perp}\right) \delta \mathbf{n}^{\prime} .
\end{aligned}
$$

The integral on the right-hand side of Eq. (B4) can be rewritten in the form

$$
\begin{aligned}
\int \frac{\mathrm{d}^{2} \mathbf{r}_{\perp}}{(2 \pi)^{2}} \exp \left(-i \mathbf{k}_{\perp} \cdot \mathbf{r}_{\perp}\right)\left(\hat{\mathbf{z}} \cdot \nabla_{\perp} \times \mathbf{B}_{\perp}\right) \delta \mathbf{n}^{\prime} \\
\quad=\int \mathrm{d}^{2} \mathbf{k}_{\perp}{ }^{\prime} \delta \widetilde{\mathbf{n}}^{\prime}\left(\mathbf{k}_{\perp}-\mathbf{k}_{\perp}{ }^{\prime}, z\right) \int \frac{\mathrm{d}^{2} \mathbf{r}_{\perp}}{(2 \pi)^{2}} \\
\quad \exp \left(-i \mathbf{k}_{\perp}{ }^{\prime} \cdot \mathbf{r}_{\perp}\right)\left(\hat{\mathbf{z}} \cdot \nabla_{\perp} \times \mathbf{B}_{\perp}\right) \\
=i \int \mathrm{d}^{2} \mathbf{k}_{\perp}{ }^{\prime} \delta \widetilde{\mathbf{n}}^{\prime}\left(\mathbf{k}_{\perp}-\mathbf{k}_{\perp}{ }^{\prime}, z\right)\left[\hat{\mathbf{z}} \cdot \mathbf{k}_{\perp}{ }^{\prime} \times \widetilde{\mathbf{B}}_{\perp}\left(\mathbf{k}_{\perp}{ }^{\prime}, z\right)\right] \\
=i \int \mathrm{d}^{2} \mathbf{k}_{\perp}{ }^{\prime}\left[\delta \widetilde{\mathbf{n}}^{\prime}\left(\mathbf{k}_{\perp}-\mathbf{k}_{\perp}{ }^{\prime}, z\right): \mathbf{k}_{\perp}{ }^{\prime}\right]: \boldsymbol{\sigma}^{+}: \widetilde{\mathbf{B}}_{\perp}\left(\mathbf{k}_{\perp}{ }^{\prime}, z\right) .
\end{aligned}
$$

Inserting Eq. (B5) into Eq. (B4) and multiplying the resultant equation by matrix $\overleftrightarrow{\boldsymbol{\sigma}}$, we obtain Eq. (18).

\section{APPENDIX C: CHANGE OF DEPENDENT VARIABLES}

By inserting Eqs. (21) into Eq. (17) we get

$$
\begin{aligned}
& \frac{i k^{2}}{\omega}\left[\frac{\partial \tilde{\mathbf{E}}_{\perp}{ }^{(+)}}{\partial z} \exp (i h z)+\frac{\partial \tilde{\mathbf{E}}_{\perp}{ }^{(-)}}{\partial z} \exp (-i h z)\right] \\
& -\frac{k^{2} h}{\omega}\left[\tilde{\mathbf{E}}_{\perp}^{(+)} \exp (i h z)-\widetilde{\mathbf{E}}_{\perp}^{\left({ }^{(-)}\right.} \exp (-i h z)\right] \\
& =-\frac{k^{2} h}{\omega}\left[\widetilde{\mathbf{E}}_{\perp}{ }^{(+)} \exp (i h z)-\widetilde{\mathbf{E}}_{\perp}^{(-)} \exp (-i h z)\right] \\
& +\frac{2 k^{2}}{\omega n_{0}} \int \mathrm{d}^{2} \mathbf{k}_{\perp}{ }^{\prime} h^{\prime} \delta n_{z z}\left(\mathbf{k}_{\perp}-\mathbf{k}_{\perp}{ }^{\prime}, z\right) \\
& \left(\mathbf{k}_{\perp}: \mathbf{k}_{\perp}{ }^{\prime}\right): \overleftrightarrow{\boldsymbol{\sigma}}: \overleftrightarrow{\Lambda}^{\prime-1}: \overleftrightarrow{\boldsymbol{\sigma}}:\left[\tilde{\mathbf{E}}_{\perp}{ }^{(+)}\left(\mathbf{k}_{\perp}{ }^{\prime}, \mathbf{z}\right)\right. \\
& \left.\exp \left(i h^{\prime} z\right)-\widetilde{\mathbf{E}}_{\perp}{ }^{(-)}\left(\mathbf{k}_{\perp}{ }^{\prime}, z\right) \exp \left(-i h^{\prime} z\right)\right] \\
& +\frac{2 k^{2}}{\omega n_{0}} \int \mathrm{d}^{2} \mathbf{k}_{\perp}{ }^{\prime}\left[\mathbf{k}_{\perp}: \delta \widetilde{\mathbf{n}}^{\prime}\left(\mathbf{k}_{\perp}-\mathbf{k}_{\perp}{ }^{\prime}, z\right)\right] \\
& :\left[\tilde{\mathbf{E}}_{\perp}{ }^{(+)}\left(\mathbf{k}_{\perp}{ }^{\prime}, z\right) \exp \left(i h^{\prime} \boldsymbol{z}\right)\right. \\
& \left.+\widetilde{\mathbf{E}}_{\perp}{ }^{(-)}\left(\mathbf{k}_{\perp}{ }^{\prime}, \mathbf{z}\right) \exp \left(-i h^{\prime} z\right)\right],
\end{aligned}
$$

from which it follows that

$$
\begin{aligned}
i\left[\frac{\partial \widetilde{\mathbf{E}}_{\perp}{ }^{(+)}}{\partial z}\right. & \left.\exp (i h z)+\frac{\partial \widetilde{\mathbf{E}}_{\perp}{ }^{(-)}}{\partial z} \exp (-i h z)\right] \\
= & \frac{2}{n_{0}} \int \mathrm{d}^{2} \mathbf{k}_{\perp}{ }^{\prime} h^{\prime} \delta n_{z z}\left(\mathbf{k}_{\perp}-\mathbf{k}_{\perp}{ }^{\prime}, z\right) \\
& \left(\mathbf{k}_{\perp}: \mathbf{k}_{\perp}{ }^{\prime}\right): \overleftrightarrow{\boldsymbol{\sigma}}: \overleftrightarrow{\boldsymbol{\Lambda}}^{\prime}{ }^{-1}: \overleftrightarrow{\boldsymbol{\sigma}}:\left[\widetilde{\mathbf{E}}_{\perp}{ }^{(+)}\right. \\
& \left(\mathbf{k}_{\perp}{ }^{\prime}, z\right) \exp \left(i h^{\prime} z\right)-\widetilde{\mathbf{E}}_{\perp}{ }^{(-)} \\
& \left.\left(\mathbf{k}_{\perp}{ }^{\prime}, z\right) \exp \left(-i h^{\prime} z\right)\right]+\frac{2}{n_{0}} \int \mathrm{d}^{2} \mathbf{k}_{\perp}{ }^{\prime}\left[\mathbf{k}_{\perp}: \delta \widetilde{\mathbf{n}}^{\prime}\left(\mathbf{k}_{\perp}\right.\right. \\
& \left.\left.-\mathbf{k}_{\perp}{ }^{\prime}, z\right)\right]:\left[\tilde{\mathbf{E}}_{\perp}{ }^{(+)}\left(\mathbf{k}_{\perp}{ }^{\prime}, z\right) \exp \left(i h^{\prime} z\right)\right. \\
& \left.+\widetilde{\mathbf{E}}_{\perp}{ }^{(-)}\left(\mathbf{k}_{\perp}{ }^{\prime}, z\right) \exp \left(-i h^{\prime} z\right)\right],
\end{aligned}
$$


where $\overleftrightarrow{\Lambda}^{\prime}$ is obtained from $\overleftrightarrow{\Lambda}$ [see Eq. (19)] by means of the substitution $\left(k_{x}, k_{y}\right) \rightarrow\left(k_{x}{ }^{\prime}, k_{y}{ }^{\prime}\right)$. By taking advantage of the relations

$$
\begin{gathered}
h^{2}=k^{2}-k_{\perp}{ }^{2}, \quad \overleftrightarrow{\boldsymbol{\Lambda}}^{-1}=\frac{1}{h^{2} k^{2}}\left(k^{2}-\mathbf{k}_{\perp}: \mathbf{k}_{\perp}\right), \\
h^{\prime}\left(\mathbf{k}_{\perp}: \mathbf{k}_{\perp}{ }^{\prime}\right): \overleftrightarrow{\boldsymbol{\sigma}}: \overleftrightarrow{\boldsymbol{\Lambda}}^{\prime}{ }^{-1}: \overleftrightarrow{\boldsymbol{\sigma}}=-\frac{\mathbf{k}_{\perp}: \mathbf{k}_{\perp}{ }^{\prime}}{h^{\prime}}
\end{gathered}
$$

we get from Eq. (C2)

$$
\begin{aligned}
& \frac{\partial \widetilde{\mathbf{E}}_{\perp}{ }^{(+)}}{\partial z} \exp (i h z)+\frac{\partial \widetilde{\mathbf{E}}_{\perp}{ }^{(-)}}{\partial z} \exp (-i h z) \\
& =\frac{2 i}{n_{0}} \int \mathrm{d}^{2} \mathbf{k}_{\perp}{ }^{\prime}\left[\delta \widetilde{n}_{z z}\left(\mathbf{k}_{\perp}-\mathbf{k}_{\perp}{ }^{\prime}, z\right) \frac{\mathbf{k}_{\perp}: \mathbf{k}_{\perp}{ }^{\prime}}{h^{\prime}}\right. \\
& \left.\quad-\mathbf{k}_{\perp}: \delta \widetilde{\mathbf{n}}^{\prime}\left(\mathbf{k}_{\perp}-\mathbf{k}_{\perp}{ }^{\prime}, z\right)\right]: \widetilde{\mathbf{E}}_{\perp}{ }^{(+)}\left(\mathbf{k}_{\perp}{ }^{\prime}, z\right) \exp \left(i h^{\prime} z\right) \\
& \quad+\int \mathrm{d}^{2} \mathbf{k}_{\perp}{ }^{\prime}\left[-\delta \widetilde{n}_{z z}\left(\mathbf{k}_{\perp}-\mathbf{k}_{\perp}{ }^{\prime}, z\right) \frac{\mathbf{k}_{\perp}: \mathbf{k}_{\perp}{ }^{\prime}}{h^{\prime}}-\mathbf{k}_{\perp}: \delta \widetilde{\mathbf{n}}^{\prime}\right. \\
& \left.\quad\left(\mathbf{k}_{\perp}-\mathbf{k}_{\perp}{ }^{\prime}, z\right)\right]: \widetilde{\mathbf{E}}_{\perp}{ }^{(-)}\left(\mathbf{k}_{\perp}{ }^{\prime}, z\right) \exp \left(-i h^{\prime} z\right) .
\end{aligned}
$$

By inserting Eqs. (21) into Eq. (18) and following an analogous procedure, we also obtain

$$
\begin{aligned}
\frac{\partial \widetilde{\mathbf{E}}_{\perp}{ }^{(+)}}{\partial z} \exp (i h z)-\frac{\partial \widetilde{\mathbf{E}}_{\perp}{ }^{(-)}}{\partial z} \exp (-i h z) \\
=\frac{2 i}{n_{0}} \int \mathrm{d}^{2} \mathbf{k}_{\perp}{ }_{\perp} \frac{\overleftrightarrow{\boldsymbol{\sigma}}: \overleftrightarrow{\Lambda}: \overleftrightarrow{\boldsymbol{\sigma}}}{h}:\left[-\delta{\widetilde{\tilde{n}_{\perp}}}_{\perp}\left(\mathbf{k}_{\perp}-\mathbf{k}_{\perp}{ }^{\prime}, z\right)\right. \\
\left.\quad+\frac{\delta \widetilde{\mathbf{n}}^{\prime}\left(\mathbf{k}_{\perp}-\mathbf{k}_{\perp}{ }^{\prime}, z\right): \mathbf{k}_{\perp}{ }^{\prime}}{h^{\prime}}\right]: \widetilde{\mathbf{E}}_{\perp}{ }^{(+)}\left(\mathbf{k}_{\perp}{ }^{\prime}, z\right) \exp \left(i h^{\prime} z\right) \\
\quad+\frac{2 i}{n_{0}} \int \mathrm{d}^{2} \mathbf{k}_{\perp}{ }^{\prime} \frac{\overleftrightarrow{\boldsymbol{\sigma}}: \overleftrightarrow{\Lambda}: \overleftrightarrow{\boldsymbol{\sigma}}}{h}:\left[-\delta{\widetilde{n_{\perp}}}_{\perp}\left(\mathbf{k}_{\perp}-\mathbf{k}_{\perp}{ }^{\prime}, z\right)\right. \\
-\frac{\left.\delta \widetilde{\mathbf{n}}^{\prime}\left(\mathbf{k}_{\perp}-\mathbf{k}_{\perp}{ }^{\prime}, z\right): \mathbf{k}_{\perp}{ }^{\prime}\right]: \widetilde{\mathbf{E}}_{\perp}{ }^{(-)}\left(\mathbf{k}_{\perp}{ }^{\prime}, z\right) \exp \left(-i h^{\prime} z\right) .}{h^{\prime}}
\end{aligned}
$$

Finally, by adding and subtracting Eqs. (C5) and (C6) we get Eqs. (22) and (23), respectively.

\section{APPENDIX D: DERIVATION OF PROPAGATION EQUATIONS IN ORDINARY} SPACE

The integral that appears on the right-hand side of Eq. (25) consists of four terms, which can be expressed, in the same order, as

$$
\begin{aligned}
& \int \mathrm{d}^{2} \mathbf{k}_{\perp} \exp \left(i \mathbf{k}_{\perp} \cdot \mathbf{r}_{\perp}\right) \int \mathrm{d}^{2} \mathbf{k}_{\perp}{ }^{\prime} \delta \widetilde{n}_{z z}\left(\mathbf{k}_{\perp}-\mathbf{k}_{\perp}{ }^{\prime}, z\right) \\
& \frac{\mathbf{k}_{\perp}: \mathbf{k}_{\perp}{ }^{\prime}}{h^{\prime}}: \widetilde{\mathbf{E}}_{\perp}{ }^{(+)}\left(\mathbf{k}_{\perp}{ }^{\prime}, \mathbf{z}\right) \exp \left(i h^{\prime} z\right) \\
& =-\int \mathrm{d}^{2} \mathbf{k}_{\perp} \exp \left(i \mathbf{k}_{\perp} \cdot \mathbf{r}_{\perp}\right) i \mathbf{k}_{\perp} \int \mathrm{d}^{2} \mathbf{k}_{\perp}{ }^{\prime} \delta \widetilde{n}_{z z}\left(\mathbf{k}_{\perp}\right. \\
& \left.-\mathbf{k}_{\perp}{ }^{\prime}, \mathbf{z}\right) \frac{1}{h^{\prime}} i \mathbf{k}_{\perp}{ }^{\prime} \cdot \widetilde{\mathbf{E}}_{\perp}{ }^{(+)}\left(\mathbf{k}_{\perp}{ }^{\prime}, z\right) \exp \left(i h^{\prime} z\right) \\
& =-\nabla_{\perp} \int \mathrm{d}^{2} \mathbf{k}_{\perp} \exp \left(i \mathbf{k}_{\perp} \cdot \mathbf{r}_{\perp}\right) \int \mathrm{d}^{2} \mathbf{k}_{\perp}{ }^{\prime} \delta \widetilde{n}_{z z}\left(\mathbf{k}_{\perp}\right. \\
& \left.-\mathbf{k}_{\perp}{ }^{\prime}, z\right) \frac{1}{h^{\prime}} i \mathbf{k}_{\perp}{ }^{\prime} \cdot \widetilde{\mathbf{E}}_{\perp}{ }^{(+)}\left(\mathbf{k}_{\perp}{ }^{\prime}, z\right) \exp \left(i h^{\prime} z\right) \\
& =-\nabla_{\perp}\left[\delta n_{z z}\left(\mathbf{r}_{\perp}, z\right) \int \mathrm{d}^{2} \mathbf{k}_{\perp} \exp \left(i \mathbf{k}_{\perp} \cdot \mathbf{r}_{\perp}\right) \frac{1}{h} i \mathbf{k}_{\perp}\right. \\
& \left.\widetilde{\mathbf{E}}_{\perp}^{(+)}\left(\mathbf{k}_{\perp}, z\right) \exp (i h z)\right] \\
& =-\nabla_{\perp}\left[\delta n_{z z} \frac{1}{\sqrt{k^{2}+\nabla_{\perp}^{2}}} \nabla_{\perp} \cdot \int \mathrm{d}^{2} \mathbf{k}_{\perp} \exp \left(i \mathbf{k}_{\perp} \cdot \mathbf{r}_{\perp}\right)\right. \\
& \left.\tilde{\mathbf{E}}_{\perp}^{(+)}\left(\mathbf{k}_{\perp}, z\right) \exp (i h z)\right] \\
& =-\nabla_{\perp}\left[\delta n_{z z} \frac{1}{\sqrt{k^{2}+\nabla_{\perp}^{2}}}\left(\nabla_{\perp} \cdot \mathbf{E}_{\perp}{ }^{(+)}\right)\right],
\end{aligned}
$$

$$
\begin{aligned}
\int & \mathrm{d}^{2} \mathbf{k}_{\perp} \exp \left(i \mathbf{k}_{\perp} \cdot \mathbf{r}_{\perp}\right) \int \mathrm{d}^{2} \mathbf{k}_{\perp}{ }^{\prime} \\
& {\left[-\mathbf{k}_{\perp}: \delta \widetilde{\mathbf{n}}^{\prime}\left(\mathbf{k}_{\perp}-\mathbf{k}_{\perp}{ }^{\prime}, z\right)\right]: \widetilde{\mathbf{E}}_{\perp}{ }^{(+)}\left(\mathbf{k}_{\perp}{ }^{\prime}, z\right) \exp \left(i h^{\prime} z\right) } \\
= & i \int \mathrm{d}^{2} \mathbf{k}_{\perp} \exp \left(i \mathbf{k}_{\perp} \cdot \mathbf{r}_{\perp}\right) i \mathbf{k}_{\perp} \int \mathrm{d}^{2} \mathbf{k}_{\perp}{ }^{\prime} \delta \widetilde{\mathbf{n}}^{\prime}\left(\mathbf{k}_{\perp}-\mathbf{k}_{\perp}{ }^{\prime}, z\right) \\
& \quad \widetilde{\mathbf{E}}_{\perp}{ }^{(+)}\left(\mathbf{k}_{\perp}{ }^{\prime}, z\right) \exp \left(i h^{\prime} z\right) \\
= & i \nabla_{\perp} \int \mathrm{d}^{2} \mathbf{k}_{\perp} \exp \left(i \mathbf{k}_{\perp} \cdot \mathbf{r}_{\perp}\right) \int \mathrm{d}^{2} \mathbf{k}_{\perp}{ }^{\prime} \delta \widetilde{\mathbf{n}}^{\prime}\left(\mathbf{k}_{\perp}-\mathbf{k}_{\perp}{ }^{\prime}, z\right) \\
= & i \nabla_{\perp}\left(\delta \mathbf{\mathbf { n }}^{\prime} \cdot \mathbf{E}_{\perp}{ }^{(+)}\left(\mathbf{k}_{\perp}{ }^{\prime}, z\right) \exp \left(i h^{\prime} z\right),\right.
\end{aligned}
$$




$$
\begin{aligned}
& : \widetilde{\mathbf{E}}_{\perp}{ }^{(+)}\left(\mathbf{k}_{\perp}{ }^{\prime}, z\right) \exp \left(i h^{\prime} z\right) \\
& +\frac{1}{\sqrt{k^{2}+\nabla_{\perp}^{2}}} \int \mathrm{d}^{2} \mathbf{k}_{\perp} \exp \left(i \mathbf{k}_{\perp} \cdot \mathbf{r}_{\perp}\right) i \mathbf{k}_{\perp}\left[i \mathbf{k}_{\perp}\right. \\
& \cdot \int \mathrm{d}^{2} \mathbf{k}_{\perp}{ }^{\prime} \delta \widetilde{\mathbf{n}}_{\perp}\left(\mathbf{k}_{\perp}-\mathbf{k}_{\perp}{ }^{\prime}, z\right) \\
& \left.: \widetilde{E}_{\perp}{ }^{(+)}\left(\mathbf{k}_{\perp}{ }^{\prime}, z\right) \exp \left(i h^{\prime} z\right)\right] \\
& =\frac{k^{2}}{\sqrt{k^{2}+\nabla_{\perp}^{2}}}\left(\delta \overrightarrow{\mathbf{n}}_{\perp}: \mathbf{E}_{\perp}{ }^{(+)}\right)+\frac{1}{\sqrt{k^{2}+\nabla_{\perp}^{2}}} \\
& \nabla_{\perp}\left[\nabla_{\perp} \cdot \int \mathrm{d}^{2} \mathbf{k}_{\perp} \exp \left(i \mathbf{k}_{\perp} \cdot \mathbf{r}_{\perp}\right) \int \mathrm{d}^{2} \mathbf{k}_{\perp}{ }^{\prime} \delta \widetilde{\mathbf{n}}_{\perp}\right. \\
& \left.\left(\mathbf{k}_{\perp}-\mathbf{k}_{\perp}{ }^{\prime}, z\right): \widetilde{\mathbf{E}}_{\perp}{ }^{(+)}\left(\mathbf{k}_{\perp}{ }^{\prime}, z\right) \exp \left(i h^{\prime} z\right)\right] \\
& =\frac{k^{2}}{\sqrt{k^{2}+\nabla_{\perp}^{2}}}\left(\delta \overleftrightarrow{\mathbf{n}}_{\perp}: \mathbf{E}_{\perp}{ }^{(+)}\right) \\
& +\frac{1}{\sqrt{k^{2}+\nabla_{\perp}^{2}}} \nabla_{\perp}\left[\nabla_{\perp} \cdot\left(\delta \overleftrightarrow{\mathbf{n}}_{\perp}: \mathbf{E}_{\perp}^{(+)}\right)\right], \\
& \int \mathrm{d}^{2} \mathbf{k}_{\perp} \exp \left(i \mathbf{k}_{\perp} \cdot \mathbf{r}_{\perp}\right) \int \mathrm{d}^{2} \\
& \mathbf{k}_{\perp}{ }^{\prime}\left\{-\left(\frac{k^{2}-\mathbf{k}_{\perp}: \mathbf{k}_{\perp}}{h}\right):\left[\frac{\delta \widetilde{\mathbf{n}}^{\prime}\left(\mathbf{k}_{\perp}-\mathbf{k}_{\perp}{ }^{\prime}, z\right): \mathbf{k}_{\perp}{ }^{\prime}}{h^{\prime}}\right]\right\} \\
& : \widetilde{\mathbf{E}}_{\perp}{ }^{(+)}\left(\mathbf{k}_{\perp}{ }^{\prime}, \boldsymbol{z}\right) \exp \left(i h^{\prime} \boldsymbol{z}\right) \\
& =\int \mathrm{d}^{2} \mathbf{k}_{\perp} \exp \left(i \mathbf{k}_{\perp} \cdot \mathbf{r}_{\perp}\right) \int \mathrm{d}^{2} \mathbf{k}_{\perp}{ }^{\prime} \\
& {\left[-\frac{k^{2}}{h} \frac{\delta \widetilde{\mathbf{n}}^{\prime}\left(\mathbf{k}_{\perp}-\mathbf{k}_{\perp}{ }^{\prime}, z\right): \mathbf{k}_{\perp}{ }^{\prime}}{h^{\prime}}\right]} \\
& : \widetilde{\mathbf{E}}_{\perp}{ }^{(+)}\left(\mathbf{k}_{\perp}{ }^{\prime}, z\right) \\
& \exp \left(i h^{\prime} z\right)+\int \mathrm{d}^{2} \mathbf{k}_{\perp} \exp \left(i \mathbf{k}_{\perp} \cdot \mathbf{r}_{\perp}\right) \\
& \int \mathrm{d}^{2} \mathbf{k}_{\perp}{ }^{\prime}\left[\frac{\mathbf{k}_{\perp}: \mathbf{k}_{\perp}}{h}: \frac{\delta \widetilde{\mathbf{n}}^{\prime}\left(\mathbf{k}_{\perp}-\mathbf{k}_{\perp}{ }^{\prime}, z\right): \mathbf{k}_{\perp}{ }^{\prime}}{h^{\prime}}\right] \\
& : \tilde{\mathbf{E}}_{\perp}{ }^{(+)}\left(\mathbf{k}_{\perp}{ }^{\prime}, z\right) \exp \left(i h^{\prime} z\right) .
\end{aligned}
$$

The two terms that appear on the right-hand side of Eq. (D4) can in turn be expressed as

$$
\begin{aligned}
& \int \mathrm{d}^{2} \mathbf{k}_{\perp} \exp \left(i \mathbf{k}_{\perp} \cdot \mathbf{r}_{\perp}\right) \int \mathrm{d}^{2} \mathbf{k}_{\perp}{ }^{\prime} \\
& {\left[-\frac{k^{2}}{h} \frac{\delta \widetilde{\mathbf{n}}^{\prime}\left(\mathbf{k}_{\perp}-\mathbf{k}_{\perp}{ }^{\prime}, z\right): \mathbf{k}_{\perp}{ }^{\prime}}{h^{\prime}}\right]} \\
& : \tilde{\mathbf{E}}_{\perp}{ }^{(+)}\left(\mathbf{k}_{\perp}{ }^{\prime}, \mathbf{z}\right) \exp \left(i h^{\prime} \boldsymbol{z}\right) \\
& =-\frac{k^{2}}{\sqrt{k^{2}+\nabla_{\perp}^{2}}} \int \mathrm{d}^{2} \mathbf{k}_{\perp} \exp \left(i \mathbf{k}_{\perp} \cdot \mathbf{r}_{\perp}\right) \\
& \int \mathrm{d}^{2} \mathbf{k}_{\perp}{ }^{\prime} \delta \widetilde{\mathbf{n}}^{\prime}\left(\mathbf{k}_{\perp}-\mathbf{k}_{\perp}{ }^{\prime}, z\right) \\
& \frac{\mathbf{k}_{\perp}{ }^{\prime} \cdot \widetilde{\mathbf{E}}_{\perp}{ }^{(+)}\left(\mathbf{k}_{\perp}{ }^{\prime}, z\right)}{h^{\prime}} \exp \left(i h^{\prime} z\right) \\
& =\frac{i k^{2}}{\sqrt{k^{2}+\nabla_{\perp}^{2}}}\left[\delta \mathbf{n}^{\prime} \frac{1}{\sqrt{k^{2}+\nabla_{\perp}^{2}}}\right. \\
& \int \mathrm{d}^{2} \mathbf{k}_{\perp} \exp \left(i \mathbf{k}_{\perp} \cdot \mathbf{r}_{\perp}\right) i \mathbf{k}_{\perp} \\
& \left.\widetilde{\mathbf{E}}_{\perp}^{(+)}\left(\mathbf{k}_{\perp}, \mathbf{z}\right) \exp (i h z)\right] \\
& =\frac{i k^{2}}{\sqrt{k^{2}+\nabla_{\perp}^{2}}}\left[\delta \mathbf{n}^{\prime} \frac{1}{\sqrt{k^{2}+\nabla_{\perp}^{2}}} \nabla_{\perp}\right. \\
& \left.\cdot \int \mathrm{d}^{2} \mathbf{k}_{\perp} \exp \left(i \mathbf{k}_{\perp} \cdot \mathbf{r}_{\perp}\right) \widetilde{\mathbf{E}}_{\perp}{ }^{(+)}\left(\mathbf{k}_{\perp}, z\right) \exp (i h z)\right] \\
& =\frac{i k^{2}}{\sqrt{k^{2}+\nabla_{\perp}^{2}}}\left[\delta \mathbf{n}^{\prime} \frac{1}{\sqrt{k^{2}+\nabla_{\perp}^{2}}}\left(\nabla_{\perp} \cdot \mathbf{E}_{\perp}^{(+)}\right)\right],
\end{aligned}
$$$$
\int \mathrm{d}^{2} \mathbf{k}_{\perp} \exp \left(i \mathbf{k}_{\perp} \cdot \mathbf{r}_{\perp}\right)
$$$$
\int \mathrm{d}^{2} \mathbf{k}_{\perp}{ }^{\prime}\left[\frac{\mathbf{k}_{\perp}: \mathbf{k}_{\perp}}{h}: \frac{\delta \widetilde{\mathbf{n}}^{\prime}\left(\mathbf{k}_{\perp}-\mathbf{k}_{\perp}{ }^{\prime}, \mathbf{z}\right): \mathbf{k}_{\perp}{ }^{\prime}}{h^{\prime}}\right]
$$$$
: \tilde{\mathbf{E}}_{\perp}{ }^{(+)}\left(\mathbf{k}_{\perp}{ }^{\prime}, \mathbf{z}\right) \exp \left(i h^{\prime} z\right)
$$$$
=-\frac{1}{\sqrt{k^{2}+\nabla_{\perp}^{2}}} \int \mathrm{d}^{2} \mathbf{k}_{\perp} \exp \left(i \mathbf{k}_{\perp} \cdot \mathbf{r}_{\perp}\right)
$$$$
i \mathbf{k}_{\perp}\left[i \mathbf{k}_{\perp} \cdot \int \mathrm{d}^{2} \mathbf{k}_{\perp}{ }^{\prime} \delta \widetilde{\mathbf{n}}^{\prime}\left(\mathbf{k}_{\perp}-\mathbf{k}_{\perp}{ }^{\prime}, z\right)\right.
$$$$
\left.\frac{\mathbf{k}_{\perp}{ }^{\prime} \cdot \widetilde{\mathbf{E}}_{\perp}{ }^{(+)}\left(\mathbf{k}_{\perp}{ }^{\prime}, z\right)}{h^{\prime}} \exp \left(i h^{\prime} z\right)\right]
$$$$
=-\frac{1}{\sqrt{k^{2}+\nabla_{\perp}^{2}}} \nabla_{\perp}\left[\nabla_{\perp} \cdot \int \mathrm{d}^{2} \mathbf{k}_{\perp} \exp \left(i \mathbf{k}_{\perp} \cdot \mathbf{r}_{\perp}\right)\right.
$$$$
\int \mathrm{d}^{2} \mathbf{k}_{\perp}{ }^{\prime} \delta \widetilde{\mathbf{n}}^{\prime}\left(\mathbf{k}_{\perp}-\mathbf{k}_{\perp}{ }^{\prime}, z\right)
$$$$
\left.\frac{\mathbf{k}_{\perp}{ }^{\prime} \cdot \widetilde{\mathbf{E}}_{\perp}{ }^{(+)}\left(\mathbf{k}_{\perp}{ }^{\prime}, z\right)}{h^{\prime}} \exp \left(i h^{\prime} z\right)\right]
$$ 


$$
\begin{aligned}
& =\frac{i}{\sqrt{k^{2}+\nabla_{\perp}^{2}}} \nabla_{\perp}\left\{\nabla _ { \perp } \cdot \left[\delta \mathbf{n}^{\prime} \int \mathrm{d}^{2} \mathbf{k}_{\perp}\right.\right. \\
& \left.\left.\exp \left(i \mathbf{k}_{\perp} \cdot \mathbf{r}_{\perp}\right) \frac{i \mathbf{k}_{\perp} \cdot \widetilde{\mathbf{E}}_{\perp}^{(+)}\left(\mathbf{k}_{\perp}, z\right)}{h} \exp (i h z)\right]\right\} \\
& =\frac{i}{\sqrt{k^{2}+\nabla_{\perp}^{2}}} \nabla_{\perp}\left\{\nabla _ { \perp } \cdot \left[\delta \mathbf{n}^{\prime} \frac{1}{\sqrt{k^{2}+\nabla_{\perp}^{2}}} \nabla_{\perp}\right.\right. \\
& \left.\left.\int \mathrm{d}^{2} \mathbf{k}_{\perp} \exp \left(i \mathbf{k}_{\perp} \cdot \mathbf{r}_{\perp}\right) \tilde{\mathbf{E}}_{\perp}^{(+)}\left(\mathbf{k}_{\perp}, z\right) \exp (i h z)\right]\right\} \\
& =\frac{i}{\sqrt{k^{2}+\nabla_{\perp}^{2}}} \nabla_{\perp} \\
& \left\{\nabla_{\perp} \cdot\left[\delta \mathbf{n}^{\prime} \frac{1}{\sqrt{k^{2}+\nabla_{\perp}^{2}}}\left(\nabla_{\perp} \cdot \mathbf{E}_{\perp}^{(+)}\right)\right]\right\} .
\end{aligned}
$$

\section{APPENDIX E: COUPLED SYSTEM OF FORWARD AND BACKWARD PROPAGATING FIELDS}

If we follow, starting from Eqs. (22) and (23), a procedure completely analogous to that followed in Appendix D, we can derive the set of coupled equations that describes propagation of both $\widetilde{\mathbf{E}}_{\perp}{ }^{(+)}$and $\widetilde{\mathbf{E}}_{\perp}{ }^{(-)}$, which reads as

$$
\begin{aligned}
& \left(i \frac{\partial}{\partial z}+\sqrt{k^{2}+\nabla_{\perp}^{2}}\right) \mathbf{E}_{\perp}{ }^{( \pm)} \\
& =\mp \frac{k^{2}}{n_{0}} \frac{1}{\sqrt{k^{2}+\nabla_{\perp}^{2}}}\left[\delta \overleftrightarrow{\mathbf{n}}_{\perp}:\left(\mathbf{E}_{\perp}{ }^{(+)}+\mathbf{E}_{\perp}{ }^{(-)}\right)\right] \\
& +\frac{1}{n_{0}} \nabla_{\perp}\left\{\delta n_{z z} \frac{1}{\sqrt{k^{2}+\nabla_{\perp}^{2}}}\left[\nabla_{\perp} \cdot\left(\mathbf{E}_{\perp}^{(+)}-\mathbf{E}_{\perp}^{(-)}\right)\right]\right\} \\
& \mp \frac{1}{n_{0}} \frac{1}{\sqrt{k^{2}+\nabla_{\perp}^{2}}} \nabla_{\perp}\left\{\nabla_{\perp} \cdot\left[\delta \overleftrightarrow{\mathbf{n}}_{\perp}:\left(\mathbf{E}_{\perp}{ }^{(+)}+\mathbf{E}_{\perp}{ }^{(-)}\right)\right]\right\} \\
& -\frac{i}{n_{0}} \nabla_{\perp}\left[\delta \mathbf{n}^{\prime} \cdot\left(\mathbf{E}_{\perp}{ }^{(+)}+\mathbf{E}_{\perp}{ }^{(-)}\right)\right] \\
& \mp \frac{i k^{2}}{n_{0}} \frac{1}{\sqrt{k^{2}+\nabla_{\perp}^{2}}}\left\{\delta \mathbf { n } ^ { \prime } \frac { 1 } { \sqrt { k ^ { 2 } + \nabla _ { \perp } ^ { 2 } } } \left[\nabla _ { \perp } \cdot \left(\mathbf{E}_{\perp}{ }^{(+)}\right.\right.\right. \\
& \left.\left.\left.-\mathbf{E}_{\perp}^{(-)}\right)\right]\right\} \mp \frac{i}{n_{0}} \frac{1}{\sqrt{k^{2}+\nabla_{\perp}^{2}}} \nabla_{\perp} \\
& \left(\nabla_{\perp} \cdot\left\{\delta \mathbf{n}^{\prime} \frac{1}{\sqrt{k^{2}+\nabla_{\perp}^{2}}}\left[\nabla_{\perp} \cdot\left(\mathbf{E}_{\perp}{ }^{(+)}-\mathbf{E}_{\perp}{ }^{(-)}\right)\right]\right\}\right) .
\end{aligned}
$$

APPENDIX F: LONGITUDINAL COMPONENT OF THE ELECTRIC FIELD IN TERMS OF THE TRANSVERSE PART

\section{ALONE}

After we write

$$
\overleftrightarrow{\boldsymbol{\varepsilon}}=\left[n_{0}+\delta \overleftrightarrow{\mathbf{n}}(\mathbf{r})\right]^{2} \cong n_{0}^{2}+2 n_{0} \delta \overleftrightarrow{\mathbf{n}}(\mathbf{r}),
$$

Eq. (10) yields approximately

$$
\mathbf{E}_{z}=\frac{i c^{2}}{\omega}\left(\frac{1}{n_{0}{ }^{2}}-\frac{2}{n_{0}{ }^{3}} \delta n_{z z}\right) \nabla_{\perp} \times \mathbf{B}_{\perp}-\frac{2}{n_{0}}\left(\delta \mathbf{n}^{\prime} \cdot \mathbf{E}_{\perp}\right) \hat{\mathbf{z}} .
$$

We need now to express $\mathbf{B}_{\perp}$ in terms of $\mathbf{E}_{\perp}$ or, because we are considering a forward traveling field, $\mathbf{B}_{\perp}{ }^{(+)}$in terms of $\mathbf{E}_{\perp}{ }^{(+)}$. We can do this by recalling that, according to Eq. (21.1), we have

$$
\begin{aligned}
\mathbf{B}_{\perp}{ }^{(+)}\left(\mathbf{r}_{\perp}, z\right)= & \int \mathrm{d}^{2} \mathbf{k}_{\perp} \exp \left(i k_{\perp} \cdot \mathbf{r}_{\perp}\right) \widetilde{\mathbf{B}}_{\perp}{ }^{(+)}\left(\mathbf{k}_{\perp}, z\right) \\
= & \int \mathrm{d}^{2} \mathbf{k}_{\perp} \exp \left(i k_{\perp} \cdot \mathbf{r}_{\perp}\right) \frac{k^{2} h}{\omega} \overleftrightarrow{\boldsymbol{\Lambda}}^{-1}: \overleftrightarrow{\boldsymbol{\sigma}}: \\
& \widetilde{\mathbf{E}}_{\perp}{ }^{(+)}\left(\mathbf{k}_{\perp}, z\right) \exp (i h z),
\end{aligned}
$$

which yields

$$
\begin{aligned}
& \mathbf{B}_{\perp}{ }^{(+)}\left(\mathbf{r}_{\perp}, \mathbf{z}\right)=\int \mathrm{d}^{2} \mathbf{k}_{\perp} \exp \left(i \mathbf{k}_{\perp} \cdot \mathbf{r}_{\perp}\right) \frac{1}{\omega h}\left(k^{2}-\mathbf{k}_{\perp}: \mathbf{k}_{\perp}\right) \overleftrightarrow{\sigma} \\
& : \widetilde{\mathbf{E}}_{\perp}{ }^{(+)}\left(\mathbf{k}_{\perp}, z\right) \exp (i h z) \\
& =\frac{k^{2}}{\omega} \frac{1}{\sqrt{k^{2}+\nabla_{\perp}^{2}}} \\
& {\left[\overleftrightarrow{\boldsymbol{\sigma}}: \int \mathrm{d}^{2} \mathbf{k}_{\perp} \exp \left(i \mathbf{k}_{\perp} \cdot \mathbf{r}_{\perp}\right)\right.} \\
& \left.\tilde{\mathbf{E}}_{\perp}^{(+)}\left(\mathbf{k}_{\perp}, z\right) \exp (i h z)\right] \\
& +\frac{1}{\omega} \frac{1}{\sqrt{k^{2}+\nabla_{\perp}^{2}}} \int \mathrm{d}^{2} \mathbf{k}_{\perp} \\
& \exp \left(i \mathbf{k}_{\perp} \cdot \mathbf{r}_{\perp}\right) i \mathbf{k}_{\perp} i \mathbf{k}_{\perp} \\
& \cdot\left[\overleftrightarrow{\boldsymbol{\sigma}}: \tilde{\mathbf{E}}_{\perp}^{(+)}\left(\mathbf{k}_{\perp}, z\right)\right] \exp (i h z) \\
& =\frac{k^{2}}{\omega} \frac{1}{\sqrt{k^{2}+\nabla_{\perp}^{2}}}\left(\stackrel{\leftrightarrow}{\boldsymbol{\sigma}}: \mathbf{E}_{\perp}^{(+)}\right) \\
& +\frac{1}{\omega} \frac{1}{\sqrt{k^{2}+\nabla_{\perp}^{2}}}\left\{\nabla _ { \perp } \left[\nabla_{\perp} \cdot \stackrel{\leftrightarrow}{\boldsymbol{\sigma}}: \int \mathrm{d}^{2} \mathbf{k}_{\perp}\right.\right. \\
& \left.\left.\exp \left(i \mathbf{k}_{\perp} \cdot \mathbf{r}_{\perp}\right) \tilde{\mathbf{E}}_{\perp}^{(+)}\left(\mathbf{k}_{\perp}, z\right) \exp ^{(i h z)}\right]\right\} \\
& =\frac{k^{2}}{\omega} \frac{1}{\sqrt{k^{2}+\nabla_{\perp}^{2}}}\left(\overleftrightarrow{\boldsymbol{\sigma}}: \mathbf{E}_{\perp}{ }^{(+)}\right) \\
& +\frac{1}{\omega} \frac{1}{\sqrt{k^{2}+\nabla_{\perp}^{2}}}\left\{\nabla_{\perp}\left[\nabla_{\perp} \cdot\left(\overleftrightarrow{\boldsymbol{\sigma}}: \mathbf{E}_{\perp}^{(+)}\right)\right]\right\} .
\end{aligned}
$$


Because we neglected in Eq. (29) the terms that contain products of more than two $\nabla_{\perp}$, we need to keep in Eq. (F4) only the terms of zeroth order in $\nabla_{\perp}$ [see Eq. (F2)], that is,

$$
\mathbf{B}_{\perp}{ }^{(+)}\left(\mathbf{r}_{\perp}, z\right)=\frac{k}{\omega} \stackrel{\leftrightarrow}{\boldsymbol{\sigma}}: \mathbf{E}_{\perp}{ }^{(+)} .
$$

By inserting Eq. (E5) into Eq. (E2) and exploiting the relation

$$
\nabla_{\perp} \times\left(\overleftrightarrow{\boldsymbol{\sigma}}: \mathbf{E}_{\perp}^{(+)}\right)=\left(\nabla_{\perp} \cdot \mathbf{E}_{\perp}^{(+)}\right) \hat{\mathbf{z}}
$$

we finally obtain

$$
\begin{aligned}
\mathbf{E}_{z}= & \left\{\frac{i}{k}\left[1-\frac{2}{n_{0}}\left(\frac{2}{3} n_{2}\left|\mathbf{E}_{\perp}{ }^{(+)}\right|^{2}+n_{2}\left|E_{z}\right|^{2}\right)\right]\left(\nabla_{\perp} \cdot \mathbf{E}_{\perp}{ }^{(+)}\right)\right. \\
& \left.-\frac{2}{3} \frac{n_{2}}{n_{0}} E_{z}\left|\mathbf{E}_{\perp}{ }^{(+)}\right|^{2}\right\} \hat{\mathbf{z}} \cong \frac{i}{k} \nabla_{\perp} \cdot \mathbf{E}_{\perp}{ }^{(+)} \hat{\mathbf{z}} .
\end{aligned}
$$

*Permanent address, Università dell' Aquila, 67010 L'Aquila, Italy.

\section{REFERENCES}

1. M. Lax, W. H. Louisell, and W. B. McKnight, "From Maxwell to paraxial wave optics," Phys. Rev. A 11, 1365-1370 (1975).

2. See, e.g., D. Marcuse, Light Transmission Optics (Van Nostrand, New York, 1972).
3. Yu. Savchencko and B. Ya. Zel'dovich, "Wave propagation in a guiding structure: one step beyond the paraxial approximation," J. Opt. Soc. Am. B 13, 273-281 (1996).

4. M. D. Feit and J. A. Fleck, Jr., "Beam nonparaxiality, filament formation, and beam breakup in the self-focusing of optical beams," J. Opt. Soc. Am. B 5, 633-640 (1988).

5. N. Akhmediev, A. Ankiewicz, and J. M. Soto-Crespo, "Does the NLSE correctly describe beam propagation?" Opt. Lett. 18, 411-413 (1993).

6. S. Chi and Q. Guo, "Vector theory of self-focusing of an optical beam in Kerr media," Opt. Lett. 20, 1598-1600 (1995).

7. G. Fibich, "Small beam nonparaxiality arrests self-focusing of optical beams," Phys. Rev. Lett. 76, 4356-4359 (1996).

8. B. Crosignani, P. Di Porto, and A. Yariv, "Nonparaxial equation for linear and nonlinear optical propagation," Opt. Lett. 11, 778-780 (1997).

9. S. Blair and K. Wagner, " $(2+1)-D$ propagation of spatiotemporal solitary waves including higher-order corrections," Opt. Quantum Electron. 30, 697-737 (1998).

10. B. Crosignani, A. Cutolo, and P. Di Porto, "Coupled-mode theory of nonlinear propagation in multimode and singlemode fiber: envelope solitons and self-confinement," J. Opt. Soc. Am. 72, 1136-1141 (1982).

11. V. S. Liberman and B. Ya. Zel'dovich, "Birefringence by a smoothly inhomogeneous locally isotropic medium," Phys. Rev. E 49, 2389-2396 (1994).

12. A. Yu. Savchenko and B. Ya. Zel'dovich, "Birefringence by a smoothly inhomogeneous locally isotropic medium: threedimensional case," Phys. Rev. E 50, 2287-2292 (1994).

13. B. Crosignani, P. Di Porto, and A. Yariv, "Coupled-mode theory and slowly-varying approximation in guided-wave optics," Opt. Commun. 78, 237-239 (1990).

14. R. Ulrich and A. Simon, "Polarization optics of twisted single-mode fibers," Appl. Opt. 18, 2241-2251 (1979). 